\title{
WEIRD: Wide-orbit Exoplanet Search with InfraRed Direct Imaging
}

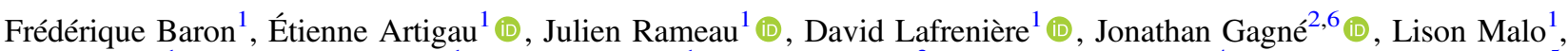 \\ Loïc Albert $^{1}$ (D), Marie-Eve Naud ${ }^{1}$ (D), René Doyon ${ }^{1}$, Markus Janson ${ }^{3}$ (D), Philippe Delorme ${ }^{4}$, and Charles Beichman ${ }^{5}$ \\ ${ }^{1}$ Institut de Recherche sur les Exoplanétes, Département de Physique, Université de Montréal, Montréal, QC H3C 3J7, Canada \\ ${ }^{2}$ Carnegie Institution of Washington DTM, 5241 Broad Branch Road NW, Washington, DC 20015, USA \\ ${ }^{3}$ Department of Astronomy, Stockholm University, SE-106 91 Stockholm, Sweden \\ ${ }^{4}$ Université de Grenoble Alpes, CNRS, IPAG, F-38000 Grenoble, France \\ ${ }^{5}$ NASA Exoplanet Science Institute, California Institute of Technology, Jet Propulsion Laboratory, Pasadena, CA 91125, USA \\ Received 2018 May 30; revised 2018 July 19; accepted 2018 July 22; published 2018 August 30
}

\begin{abstract}
We report results from the Wide-orbit Exoplanet search with InfraRed Direct imaging, or WEIRD, a survey designed to search for Jupiter-like companions on very wide orbits (1000-5000 au) around young stars $(<120 \mathrm{Myr})$ that are known members of moving groups in the solar neighborhood $(<70 \mathrm{pc})$. Companions that share the same age, distance, and metallicity as their host while being on large enough orbits to be studied as "isolated" objects make prime targets for spectroscopic observations, and they are valuable benchmark objects for exoplanet atmosphere models. The search strategy is based on deep imaging in multiple bands across the near-infrared domain. For all 177 objects of our sample, $z_{a b}^{\prime}, J$, [3.6], and [4.5] images were obtained with CFHT/MegaCam, GEMINI/GMOS, CFHT/WIRCam, GEMINI/Flamingos-2, and Spitzer/IRAC. Using this set of four images per target, we searched for sources with red $z_{a b}^{\prime}$ and [3.6]-[4.5] colors, typically reaching good completeness down to $2 M_{\text {Jup }}$ companions, while going down to $1 M_{\text {Jup }}$ for some targets, at separations of 1000-5000 au. The search yielded four candidate companions with the expected colors, but they were all rejected through follow-up proper motion observations. Our results constrain the occurrence of 1-13 $M_{\text {Jup }}$ planetary-mass companions on orbits with a semimajor axis between 1000 and 5000 au at less than 0.03 , with a $95 \%$ confidence level.
\end{abstract}

Key words: planets and satellites: gaseous planets - planets and satellites: detection

Supporting material: machine-readable tables

\section{Introduction}

Since the first detection of an exoplanet around a main sequence star by Mayor \& Queloz (1995), thousands of exoplanets have been discovered, revealing planetary system architectures vastly different from that of the solar system. The most successful methods to detect exoplanets are the transit and radial velocity methods, which are more effective for planets close to their host star (up to $15 \mathrm{au}$ ). The outer regions of planetary systems can be probed by direct imaging. The first detection of a giant planet by direct imaging was made in 2004, with the discovery of a $4 M_{\text {Jup }}$ planet orbiting the brown dwarf 2MASSW J1207334-393254 (Chauvin et al. 2004), and the search for directly imaged planets has continued since then.

A good number of direct imaging surveys for planetary-mass objects on wide obits were carried out in the last decade. Some targeted only low-mass stars, such as Bowler et al. (2015), Lannier et al. (2016), and Naud et al. (2017), while others surveyed higher mass stars (Vigan et al. 2012; Nielsen et al. 2013; Rameau et al. 2013) or all spectral types (Lafrenière et al. 2007a; Heinze et al. 2010; Biller et al. 2013; Chauvin et al. 2015). Bowler (2016) did a meta-analysis using data from the most complete studies that surveyed all types of star (Lafrenière et al. 2007a; Janson et al. 2011; Vigan et al. 2012; Biller et al. 2013; Janson et al. 2013; Nielsen et al. 2013; Wahhaj et al. 2013; Brandt et al. 2014; Bowler et al. 2015) using 384 stars with spectral types B2 to M6. He obtained respective overall planet occurrence rates for BA, FGK, and $\mathrm{M}$ stars of $2.88_{-2.3}^{3.7} \%,<4.1 \%$, and $<3.9 \%$ for $5-13 M_{\mathrm{Jup}}$ planets at separations of $30-300$ au.

\footnotetext{
${ }^{6}$ NASA Sagan Fellow.
}

Direct imaging surveys have typically targeted young stars, which are prime targets because their planets are still contracting and are thus warmer and brighter than their older counterparts, for a given mass. The number of known young stars near the Sun has dramatically increased in the last few years, as a result of a growing interest in young stellar moving groups (e.g., Zuckerman \& Song 2004; Torres et al. 2008). A moving group is composed of stars that were formed together less than a few hundreds of Myr ago, and therefore still share similar $U V W$ galactic velocities, enabling their identification. In recent years, a significant effort has been made to identify a large number of low-mass stars, brown dwarfs, and isolated planetary-mass objects that are members of known young moving groups (Lépine \& Simon 2009; Shkolnik et al. 2009, 2012; Schlieder et al. 2010, 2012a, 2012b; Kiss et al. 2011; Rodriguez et al. 2011; Liu et al. 2013; Malo et al. 2013, 2014; Moór et al. 2013; Rodriguez et al. 2013; Kraus et al. 2014; Riedel et al. 2014; Gagné et al. 2014; Binks et al. 2015; Gagné et al. 2015b).

Planets found on wide orbits around young stars are very interesting because they can be characterized much better than their closer-in counterparts. First, a planet bound to a wellstudied star shares some properties with it, like its age, distance from Earth, and metallicity. Furthermore, when a planet is on an large enough orbit, it can be studied as if it were an isolated object, i.e., without adaptive optics (Naud et al. 2014; Gauza et al. 2015), and a very high-resolution spectrum can then be acquired, which is very hard to obtain for closer-in planets. Also, the large separation to the host enables direct studies that are very challenging with high-contrast imaging (e.g., accurate spectrophotometry, intermediate-resolution spectroscopy, optical 
Table 1

Young Moving Groups

\begin{tabular}{lcccc}
\hline \hline Name & $\begin{array}{c}\text { Distance } \\
(\mathrm{pc})\end{array}$ & $\begin{array}{c}\text { Age } \\
(\mathrm{Myr})\end{array}$ & Number of Members Detected & References \\
\hline $\mathrm{N} \beta$-Pictoris & $9-73$ & $24 \pm 3$ & 51 & Shkolnik et al. (2017) \\
\hline AB-Doradus & $37-77$ & $149_{-19}^{+51}$ & 58 & Bell et al. (2016) \\
\hline Argus & $8-68$ & $30-50$ & 6 & Torres et al. (2008) \\
\hline Carina & $46-88$ & $45_{-7}^{+11}$ & 15 & Bell et al. (2016) \\
\hline Columba & $42_{-4}^{+6}$ & 50 & Bell et al. (2016) \\
\hline Tucana-Horologium & $35-81$ & $45 \pm 3$ & 16 & Bell et al. (2016) \\
\hline TW Hydrae & $36-71$ & $10 \pm 3$ & Bell et al. (2016) \\
\hline
\end{tabular}

imaging, time-variability). Such planetary-mass objects are also prime targets for JWST follow-up.

Widely separated systems are of further interest because they challenge formation processes. Theories predict that giant planets form either by core accretion or gravitational instability, or else by cloud fragmentation like brown dwarfs. The former process describes a way of forming planets by first building a 5-20 $M_{\text {Earth }}$ core of rocks and ices in a protoplanetary disk (Pollack et al. 1996; Inaba et al. 2003; Alibert et al. 2009). The core then accretes gas very rapidly to form a giant planet. This method explains very well the formation of planets on close-in orbit ( $<10$ au, Mordasini et al. 2012), but struggles to explain the formation of planets on wide orbits. The second process suggests that planets form from the fragmentation of a gravitationally unstable disk (Boss 2011), which forms clumps that then can accrete gas and dust to become planets (Stamatellos et al. 2007; Bate 2012). However, this mechanism also has difficulties forming planets on wide orbits, as shown by Nayakshin (2017) and Vigan et al. (2017), for example. The last process predicts that planets on wide orbits form from the direct collapse of a molecular cloud (Padoan \& Nordlund 2004). A fragment having mass equal to a few Jupiters is formed; it then accretes gas from the cloud to form a higher-mass object. However, Bate et al. (2002) and Bate (2012) have shown that the accretion process can be stopped at a low mass if the companion is ejected away from the dense part of the envelope or if the envelope is depleted at the formation time. However, this formation process tends to form preferentially equal mass binaries and does not seem to produce systems with the high mass ratios needed to match the observed planetary systems at wide separations. Dynamical instabilities are a tantalizing alternative to explain the detected planets at large separations (Chatterjee et al. 2008; Veras et al. 2009; Baruteau \& Masset 2013). Mutual gravitational perturbations and close encounters among the planets occur and pump the eccentricity and the semimajor axis of the less massive giant planets up to 100-100,000 au (Veras et al. 2009), but close-in scatterers are yet to be discovered (Bryan et al. 2016).

We report here the results from the Wide-orbit Exoplanet search with InfraRed Direct imaging (WEIRD). The WEIRD survey started in 2014 with the goal of detecting Jupiter-like companions on very wide orbits (at separations 1000-5000 au) around all the known members of young moving groups within $70 \mathrm{pc}$. We gathered a large data set in an effort to construct the SED of such objects through of deep [3.6] and [4.5] imaging from Spitzer/ IRAC and deep seeing-limited $J$ and $z_{a b}^{\prime}$ imaging from CFHT and Gemini-South of all 177 known (at the time) young ( $<120 \mathrm{Myr}$ ) objects in a volume-limited sample of $70 \mathrm{pc}$ of the Sun. Using these data, planetary companions can be revealed through their distinctively red $z_{a b}^{\prime}-J$ and [3.6]-[4.5] colors. The range of separations studied here has barely been probed by previous direct imaging surveys, as they were limited by the field of view of highcontrast imagers - with the exception of Naud et al. (2017), which was much less sensitive than the present survey, and limited to low-mass stars. The selection of the sample of young stars and the observing strategy and data reduction are described in Section 2. Section 3 presents the results of our search, while Section 4 discusses our statistical analysis of the survey.

\section{Sample and Observations}

\subsection{Sample}

The best targets to find giant planets on very wide orbits are young stars in the solar neighborhood, because giant planets are more luminous when they are younger and they become fainter with time. Therefore, observations of younger stars are sensitive to lower-mass planets. A sample was thus created by selecting all stars within $70 \mathrm{pc}$ that are members of the following young moving groups or associations (see Table 1): TW Hydrae (de la Reza et al. 1989; Kastner et al. 1997), $\beta$ Pictoris (Zuckerman et al. 2001a), AB Doradus (Zuckerman et al. 2004), Tucana Horologium (Torres et al. 2000; Zuckerman et al. 2001b), Carina (Torres et al. 2008), Columba (Torres et al. 2008), and Argus (Makarov \& Urban 2000). The members of these groups have ages in the range $10-150 \mathrm{Myr}$. The age of the Argus moving group is not constrained well, likely because current membership lists suffer from significant contamination from unrelated field-aged stars (Bell et al. 2016). To be considered bona fide members of one group and included in our sample, the stars must have a trigonometric parallax and a radial velocity measurement, as well as $X Y Z U V W$ values consistent with the moving group membership, along with independent signatures of youth, e.g., spectroscopic signs of low-gravity, strong X-ray or UV emission or lithium absorption (see Soderblom 2010). The sample was constructed from Gagné et al. (2014), Kiss et al. (2011), Lépine \& Simon (2009), Malo et al. (2013), Schlieder et al. (2010), Shkolnik et al. (2009, 2011, 2012), Song et al. (2003), Torres et al. (2000, 2008), Zuckerman \& Webb (2000), Zuckerman et al. (2004, 2001a), Zuckerman (2001), and Zuckerman et al. (2011). We note that these publications also proposed a larger sample of strong candidates, but some of them lacked one or more measurements to be confirmed members; these objects were not included in our sample. Our complete sample includes 177 objects. 

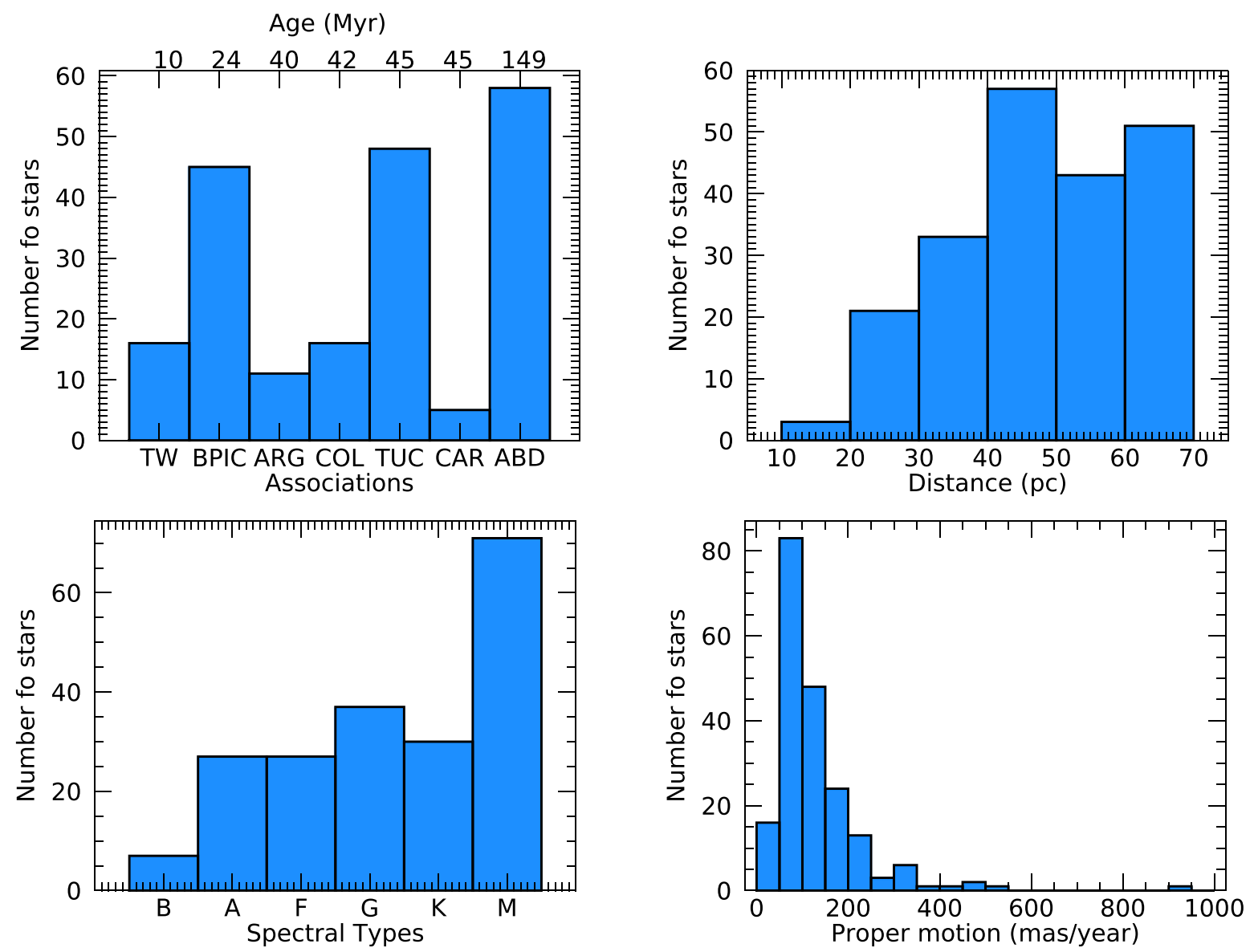

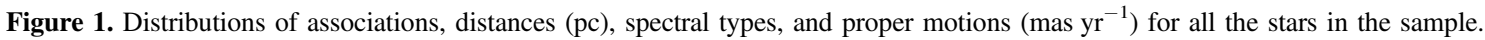

Table 2

Properties of the Sample of Bona Fide Members

\begin{tabular}{|c|c|c|c|c|c|c|c|c|}
\hline Name & $\begin{array}{c}\text { R.A. } \\
\text { (J2000.0) }\end{array}$ & $\begin{array}{c}\text { Decl. } \\
(\mathrm{J} 2000.0)\end{array}$ & $\mathrm{SpT}$ & $J$ & $H$ & $K$ & $W 1$ & $W 2$ \\
\hline HIP 490 & 000552.54 & -414511.0 & G0V & $6.464 \pm 0.011$ & $6.189 \pm 0.017$ & $6.117 \pm 0.013$ & $6.043 \pm 0.053$ & $6.053 \pm 0.023$ \\
\hline HIP 560 & 000650.08 & -230627.1 & F3V & $5.451 \pm 0.017$ & $5.331 \pm 0.045$ & $5.240 \pm 0.019$ & $5.245 \pm 0.072$ & $5.013 \pm 0.036$ \\
\hline HIP 1134 & 001410.25 & -071156.8 & F5V & $6.402 \pm 0.015$ & $6.170 \pm 0.035$ & $6.073 \pm 0.015$ & $6.049 \pm 0.046$ & $5.999 \pm 0.024$ \\
\hline HIP 1481 & 001826.12 & -632839.0 & F8V & $6.462 \pm 0.007$ & $6.248 \pm 0.033$ & $6.149 \pm 0.009$ & $6.141 \pm 0.048$ & $6.102 \pm 0.023$ \\
\hline
\end{tabular}

(This table is available in its entirety in machine-readable form.)

Multiple systems were not excluded from the sample as the presence of a lower- or equal-mass object does not exclude the possibility of having a planetary-mass object on a wide orbit. For example, Ross $458(\mathrm{AB}) \mathrm{c}$ is a triple system comprising a tight M0.5+M7 binary orbited by an $11 M_{\text {Jup }}$ object (Goldman et al. 2010) and 2MASS J01033563-5515561(AB)b, a 12-14 $M_{\text {Jup }}$ object, orbits a pair of young late-M stars at 84 au(Delorme et al. 2013). Furthermore, Wang et al. (2015) have shown that stellar multiplicity does not influence the presence of planets on wide (100-2000 au) orbits in the system. In our sample of targets, 68 are multiple systems, two host brown dwarf companions (Schneider et al. 2004; Chauvin et al. 2005) and four host known planets (Chauvin et al. 2004; Marois et al. 2008; Lagrange et al. 2009; Macintosh et al. 2015).

The properties of the 177 objects in our sample is presented in Table 2. They have spectral classes in the range A-M (the majority are $\mathrm{M}$ dwarfs), are located at distances of 7-70 pc, are located all over the sky, and have relatively high proper motions (see Figure 1). The median star has a proper motion of 100 mas yr$^{-1}$, a distance of $42 \mathrm{pc}$, and an age of $45 \mathrm{Myr}$. Table 3 lists all the systems in our sample along with their radial velocities, distances, and the association they are a member of.

\subsection{Observing Strategy}

Figure 2 presents the typical $z_{a b}^{\prime}-J$ and [3.6]-[4.5] colors as a function of spectral type for objects ranging from spectral types $\mathrm{L}$ to $\mathrm{T}$, a range relevant for the companions sought here. It shows that both $\mathrm{L}$ and $\mathrm{T}$ dwarfs have very red $z_{a b}^{\prime}-J$ colors, with the color of an L dwarf being between 2.5 and 3 mag, and the color of a T dwarf between 3 and 4.5 mag. Beyond those types, as shown by Lodieu et al. (2013), the $z_{a b}^{\prime}-J$ colors of 

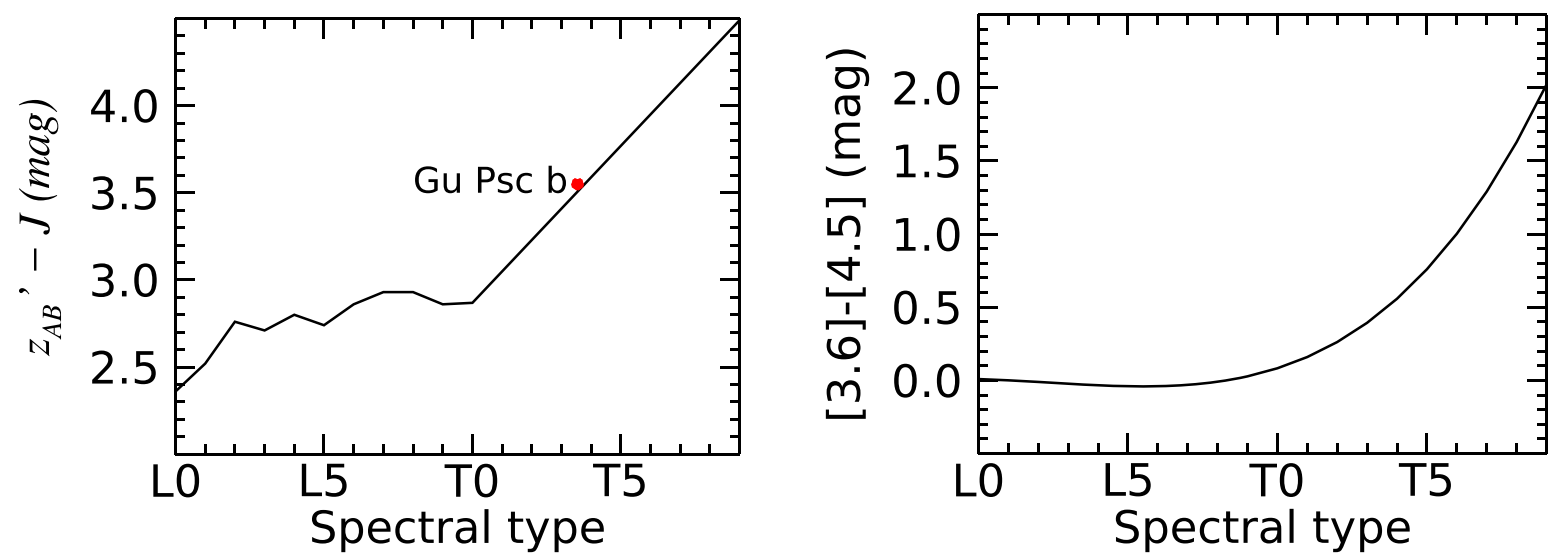

Figure 2. On the left, $z_{a b}^{\prime}-J$ vs. spectral type for $\mathrm{L}$ to T dwarfs; L dwarfs are from Hawley et al. (2002), and T dwarfs are from Albert et al. (2011). The $\mathrm{L}$ to $\mathrm{T}$ dwarfs are characterized by red $z_{a b}^{\prime}-J$ colors. The red dot represents Gu Psc b, the planetary mass object discovered by Naud et al. (2014), representative of the kind of objects we are seeking in this work. On the right, [3.6]-[4.5] for L to T dwarfs from Dupuy \& Liu (2012). We see that late-T dwarfs can be identified both by their red [3.6]-[4.5] $>1.5$ and $z_{a b}^{\prime}-J>4$ colors.

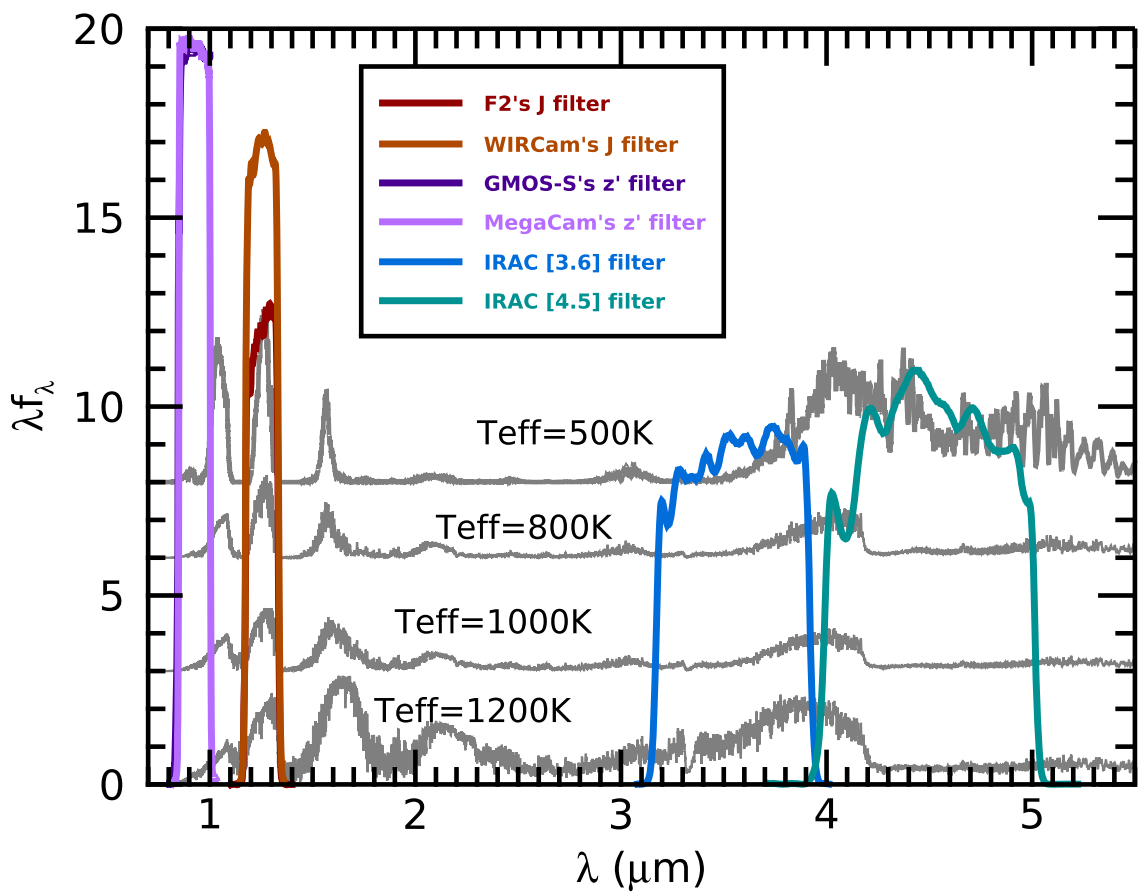

Figure 3. BT-Settl spectral energy distribution of young objects ( $\log g=4$ and solar metallicity) with effective temperatures of 500, 800, 1000, and $1200 \mathrm{~K}$. The transmission functions of the four bandpasses used for our observations $\left(z_{a b}^{\prime}, J,[3.6]\right.$, and [4.5]) are overlaid. These bandpasses provide distinctive red colors while maintaining a high flux level across the temperature range.

Table 3

Properties of the Sample of Bona Fide Members

\begin{tabular}{|c|c|c|c|c|c|}
\hline Name & $\begin{array}{c}\mu_{\alpha} \cos \delta \\
\left(\operatorname{mas~yr}^{-1}\right)\end{array}$ & $\begin{array}{c}\mu_{\delta} \\
\left(\operatorname{mas~yr}^{-1}\right)\end{array}$ & $\begin{array}{l}\text { Radial Velocities } \\
\qquad\left(\mathrm{km} \mathrm{s}^{-1}\right)\end{array}$ & $\begin{array}{l}\text { Distance } \\
(\mathrm{pc})\end{array}$ & Association \\
\hline HIP 490 & $97.53 \pm 0.38$ & $-76.27 \pm 0.44$ & $1.5 \pm 1.2$ & $39.38 \pm 0.91$ & Tucana-Horologium \\
\hline HIP 560 & $97.81 \pm 0.42$ & $-47.12 \pm 0.21$ & $6.5 \pm 3.5$ & $39.38 \pm 0.58$ & $\beta$-Pictoris \\
\hline HIP 1134 & $102.79 \pm 0.78$ & $-66.36 \pm 0.36$ & $-2.2 \pm 1.2$ & $47.14 \pm 1.42$ & Columba \\
\hline HIP 1481 & $89.37 \pm 0.48$ & $-59.46 \pm 0.50$ & $6.4 \pm 0.4$ & $41.54 \pm 0.89$ & Tucana-Horologium \\
\hline
\end{tabular}

(This table is available in its entirety in machine-readable form.)

$\mathrm{Y}$ dwarfs remain red but vary much more, ranging from 2.5 to 5 mag. In the mid-infrared, starting at around T0, the [3.6]-[4.5] color becomes increasingly red with spectral type, reaching values larger than $1.5 \mathrm{mag}$ for late-Ts. Young objects, with larger radii and correspondingly lower surface gravities, would have slightly redder colors compared to the colors of field 
dwarfs shown in the figure. The strategy used in the current survey builds on these markedly red colors of very late-type dwarfs across these four spectral bands, which enables them to be distinguished easily from earlier-type objects and most other astrophysical sources. In addition, as shown in Figure 3, these bands are also optimal to maximize the flux of the objects sought over the temperature range of interest.

The ground-based component of our survey is optimized to find companions up to spectral type $\sim \mathrm{T} 9$, while the Spitzer component is optimized for later types. At a distance of $42 \mathrm{pc}$ (the median distance of our sample), the expected $J$ magnitude of a T9 dwarf is about 21 mag. We thus designed our observations in the $J$-band to reach $21 \mathrm{mag}$. As $\mathrm{T}$ dwarfs later than T0 are expected to have $z_{a b}^{\prime}-J>3 \mathrm{mag}$, we designed our observations to reach $z_{a b}^{\prime}=24$ mag so they can be identified either through detection in both bands or as $z_{a b}^{\prime}$ dropouts. For this part of the survey, we used the same detection criteria as for the CFBDSIR survey (Delorme et al. 2008, 2010; Albert et al. 2011). That survey was a wide-field search for $\mathrm{T}$ dwarfs and early-type $\mathrm{Y}$ dwarfs, and the candidates were identified through their very red $z_{a b}^{\prime}-J>3$ mag colors if they were detected in both bands, or through $z_{a b}^{\prime}$ dropouts. The CFBDSIR survey returned only 64 candidates over the 280 square degrees observed, of which 17 were actual field $\mathrm{T}$ dwarfs. The strategy of searching for very low-mass objects using NIR colors has also been employed by the PSYM-wide survey (Naud et al. 2017) to probe nearby young M dwarfs for planetary-mass companions. The survey discovered a new planetary-mass object (9-13 $M_{\text {Jup }}$ ) orbiting at 2000 au around the M3V star Gu Psc, a highly probable member of the $\mathrm{AB}$ Doradus moving group (Naud et al. 2014). ${ }^{7}$

The Spitzer/IRAC observations were designed so that they reach a depth sufficient to identify point-sources in the field down to $\sim 0.5 \mathrm{mag}$ of the confusion limit and have their color measured accurately enough to identify them at $>5 \sigma$ level compared to the bulk of background objects. We perform the point-source detection in [3.6], which provides deeper images for flat-spectrum sources, and we use the [4.5] photometry to constrain colors. Our observations are designed to reach depths of $21.2 \mathrm{mag}(5 \sigma)$ and $20.7 \mathrm{mag}(3 \sigma)$ in [3.6] and [4.5], respectively. Planetary-mass objects close to the detection limit, with masses below 3-5 $M_{\text {Jup }}$ will have [3.6]-[4.5] $>2$ mag (see right-hand side Figure 2) and will therefore be detected at a higher signal-to-noise ratio in [4.5]. Because they would have $M_{J}>18 \mathrm{mag}$ (or $J>21 \mathrm{mag}$ for a typical target) or a spectral type $\gtrsim \mathrm{T} 8.5$, such objects would be $z_{a b^{-}}^{\prime}$ and $J$-band dropouts $\left(z_{a b}^{\prime}-[4.5]>6 \mathrm{mag}\right.$ and $\left.J-[4.5]>3.5 \mathrm{mag}\right)$. Given that background objects typically have [3.6]$[4.5] \sim 0.0 \pm 0.4 \mathrm{mag}$, such planetary-mass objects will differ from the bulk of background objects at the $>5 \sigma$-level. However, this part of the data set by itself is insensitive to more massive (>3-5 $M_{\text {Jup }}$ ) companions, as their colors don't differ enough from those of background objects.

\subsection{Observations and Data Reduction}

All targets in our sample were observed with deep seeinglimited $J$ and $z_{a b}^{\prime}$ imaging at either the Canada-France-Hawaii Telescope (CFHT) with WIRCam (Puget et al. 2004) and

\footnotetext{
Using the parallax of $21.0019 \pm 0.0721$ mas (Gaia Collaboration et al. 2018; Lindegren et al. 2018) for GU Psc from the Gaia DR2 release, along with the web tool BANYAN $\Sigma$ from Gagné et al. (2018b), we infer that the probability of $\mathrm{Gu}$ Psc to be a member of the AB Doradus moving group is $99.1 \%$, which confirms the star's membership.
}

Megacam (Boulade et al. 1998), or at Gemini-South with GMOS-S (Hook et al. 2004; Gimeno et al. 2016) and Flamingos-2 (Eikenberry et al. 2012), as well as with Spitzer/IRAC (Fazio et al. 2004) in the [3.6] and [4.5] bands. Stars with a decl. $<-35^{\circ}$ were observed from the ground at the Gemini-South Observatory while the others were observed at the CFHT. Throughout this work, all the $J$-band magnitudes are in the Vega system while all the $z_{a b}^{\prime}$ magnitudes are in the AB system. For a median star in our sample with a distance of about $42 \mathrm{pc}$ and an age of $45 \mathrm{Myr}$, the limiting magnitude in both bands corresponds to $M_{J}=17.9 \mathrm{mag}$ and $M_{z}=20.9 \mathrm{mag}$, or to an effective temperature of about $385 \mathrm{~K}$ according to models from Baraffe et al. (2003).

\subsubsection{Gemini Observations}

The observations were made from 2014 to 2017 at GeminiSouth (GS-2014B-Q-2, GS-2015A-Q-71, GS-2015B-Q-57, GS2016A-Q-69, GS-2016B-Q-33, GS-2017A-Q-58, PI Frederique Baron). We obtained deep imaging of our southern sub-sample with Flamingos-2 with the $J$ filter (J_G0802, $1.255 \mu \mathrm{m})$ and the Gemini Multi-Object Spectrograph (GMOS) in the $z_{a b}^{\prime}$ filter (z_G0328, >848 $\mu \mathrm{m}$ ). Objects beyond $30 \mathrm{pc}$, the vast majority of our targets, are sufficiently far for the entire projected $5000 \mathrm{au}$ sphere around them to fit within the GMOS/F2 FOV.

Flamingos-2 is a near-infrared wide-field imager and multiobject spectrometer with a $6.19 \operatorname{arcmin}^{2}$ circular field of view and a 0 "' 18 pixel scale. We obtained at least $600 \mathrm{~s}$ of integration time on each target, divided into a different number of expositions (at least nine) depending on the magnitude of the star and the observing conditions. A small random dither pattern was used to mitigate detector defects. The exposition time was selected to reach a limiting magnitude of $J=21 \mathrm{mag}$ at a $7 \sigma$ level. Each observation was about 20 minutes long, including all overheads.

GMOS has three $2048 \times 4608$ CCDs; when combined, they have a field of view of $5.5 \times 5.5 \mathrm{arcmin}^{2}$ and a pixel scale of 0 ". 073 . We obtained eight exposures of $65 \mathrm{~s}$ for each target of the sample. A dither pattern of $17^{\prime \prime}$ was used for all observations. The exposure time was chosen to reach a limiting magnitude of $z=24 \mathrm{mag}$ at $3 \sigma$. The observations were each about 20 minutes long, including all overheads.

The $J$-band images from F2 were reduced using a custom IDL pipeline. The individual images were reduced by subtracting dark images, dividing by flat-field images, and correcting the residual gradient from the vignetting of the Peripheral Wavefront Sensor (PWFS). This step was done by first normalizing the image to its median value, then masking regions with values significantly over the median to get rid of the stars. This image was in turn used to create a gradient image where each pixel is the median of a $128 \times 128$ pixel box of the masked image. A polynomial fit of degree 3 was then applied to the gradient image. This was divided from the F2 image to correct for the vignetting by the PWFS. The astrometric correction was then computed by anchoring the star positions on the Gaia DR1 catalog (Gaia Collaboration et al. 2016). A radial profile about the bright target star was then subtracted to help search for sources at smaller separations. Finally, a low-pass filter was created by median binning the image by $4 \times 4$ pixels, applying a $15 \times 15$ pixel median filter, and then resampling at the original image size. This low-pass filter was subtracted from the image to facilitate the detection of point sources. The individual images for a given target were then combined by taking their median, after astrometric registration, to produce the final $J$-band image. 
For GMOS, the images were also reduced using a custom IDL reduction pipeline. Each CCD was first processed separately. First, a sky correction was applied by subtracting the median of all images taken on a given night. When needed, any detector region affected by the on-instrument wave front sensor was masked. Most of the time, the wave front sensor was off the detector, but sometimes it was not possible to find a guide star outside of the FOV. The astrometric solution was found for each CCD by anchoring the field to the F2 reduced image of the same target. A high-pass filter was then applied by subtracting a median-filtered image with a width of 15 pixels. A one-dimensional median filter with a width of 61 pixels was also subtracted from each line of the image to correct for the saturation banding. The three CCDs were then combined to form a complete image, to which the astrometric solution was applied again. All the images for a given target were then aligned and stacked by taking their median to get the resulting reduced image.

\subsubsection{CFHT Observations}

Deep imaging of our northern subsample was obtained at the CFHT from 2014 to 2017 using WIRCam with the $J$ filter and MegaCam with the $z_{a b}^{\prime}$ filter (14BC016, 15AC032, 15BC012, 16AC021, 16BC018, 17AC23; PI Frédérique Baron).

WIRCam (Puget et al. 2004) is a near-infrared wide-field imager with a field of view of $20 \mathrm{arcmin}^{2}$ and a pixel scale of 0 ". 3 . It uses a mosaic of four detectors with a small gap between each. We used the $J$-band $(1.253 \mu \mathrm{m})$ filter and a homemade dither pattern of $1660 \mathrm{~s}$ expositions, arranged so that the target does a small dither of $28^{\prime \prime}$ around a pixel situated $64^{\prime \prime}$ from the corner of one detector near the center of the field, for a total of $1120 \mathrm{~s}$ of on-target integration time. A different dither pattern was used to mitigate the saturation effects of stars brighter than $J=7$. In those cases, the bright target was put in a gap between quadrants at each position of the dither pattern. With a seeing between $1^{\prime \prime}$ and 1 !" 2 , the exposure time is sufficient to reach an $\mathrm{S} / \mathrm{N}=7$ at a limiting magnitude of $J \approx 21$.

MegaCam (Boulade et al. 1998) is a wide-field optical imager with a field of view of one square degree and a pixel scale of 0 !' 187 . We used the $z_{a b}^{\prime}$ filter $\left(z_{-}\right.$G0328, $\left.>848 \mu \mathrm{m}\right)$ and a dither pattern with four positions offset by $15^{\prime \prime}$, which is twice the size of the standard dither pattern. The total integration time per target varies between 311 and $476 \mathrm{~s}$. The higher integration time is for targets with a decl. in the range -35 to -30 , to accommodate the higher airmass and maintain a good $\mathrm{S} / \mathrm{N}$. With a seeing between 0 ". 55 and 0 ". 65 , this ensures an $\mathrm{S} / \mathrm{N}$ of 3 for all our $z_{a b}^{\prime}$-band observations with a limiting magnitude of $z_{a b}^{\prime}=24$.

The WIRCam images were reduced using the method described in Albert et al. (2011). First, they were preprocessed by CFHT using their "I" iwi pipeline version 2.0. Next, a lowpass filter was created by median binning the image by $4 \times 4$ pixels, applying a $5 \times 5$ pixel median filter, and then resampling at the original image size. This low-pass filter was subtracted from the image to preserve only high spatial frequencies. After this, the different images were stacked using the Bertin software suite. First, SExtractor (Bertin \& Arnouts 1996) builds a catalog of objects in each image. This catalog is in turn read by Scamp (Bertin 2010a), which also computes the astrometric and photometric solutions by anchoring on the $J$-band data of the 2MASS catalog. Swarp (Bertin 2010b) then stacks the images together.
Data from MegaCam were first processed by CFHT's Elixir pipeline. Next, the astrometric solution from each of the 40 CCDs was found by anchoring the field on the positions from the USNO-B1 catalog. A high-pass filter was applied, as before, by subtracting an image created by median binning the image by $4 \times 4$ pixels, applying a $7 \times 7$ pixel median filter, and then re-sampling at the original image size. Then the images from the different CCDs were combined to form an image of size matching that of the WIRCam images, as the field of view of MegaCam is much wider than WIRCam's. The different images of a given target obtained on a given night were then median-combined to obtain the final reduced $z_{a b}^{\prime}$ image.

\subsubsection{Spitzer/IRAC Observations}

Our complete sample was observed with Spitzer/IRAC. Nine of our targets had previously been observed with IRAC with an exposition time that meets our requirements. The others targets were observed between 2015 and 2016 (Spitzer proposal 11092) in both IRAC [3.6] and [4.5], with a total integration time of $2160 \mathrm{~s}$ in each band (per-visit total of $5221 \mathrm{~s}$ with overheads). More precisely, we used an individual exposure time of $30 \mathrm{~s}$, two exposures per dither position per band, and a 36 exposure reuleaux dither pattern.

The Spitzer/IRAC pipeline reduced images were further processed with custom IDL routines. First, the different images of a given target were oversampled on a 0.5 pixel grid and median-combined using the pipeline-provided astrometry and polynomial distortion. Then, to preserve the point-spread function (PSF) morphology orientation in the image, in view of the PSF subtraction routines to be applied, we registered all images to a common PSF rotation angle.

Further data reduction involved the subtraction of the stellar PSF to reveal embedded and close-in sources. Because the Spitzer observations were uniform, we used the Reference Differential Imaging technique to subtract the PSF from a reference library. The strategy is similar to the reanalysis of Hubble imaging data through the ALICE project (Choquet et al. 2015; Soummer et al. 2016), and to previous analysis of archival Spitzer data (Janson et al. 2015; Durkan et al. 2016).

The library of reference PSFs was created out of the newly obtained data, using the PSFs of the observed stars. Saturated stars, very crowded fields, and low-contrast $(<1)$ visual binaries were removed from the library, resulting in a total of 111 PSFs out of the 168 targets observed. Each image was registered on a common center based on the fit of a twodimensional Moffat function. It was then normalized in brightness from the flux measured in an aperture of a radius of seven pixels centered on the PSF core. Point sources were identified as $3 \sigma$ outliers from the noise (calculated with a robust sigma estimator) after an initial PSF subtraction from the median of the reference library, excluding the given image. They were subsequently masked out in the original image.

We used classical RDI to subtract stellar PSFs in each Spizter image for both filters. Because of the very large number of point sources in our deep data, advanced techniques such as LOCI or PCA (Lafrenière et al. 2007b; Soummer et al. 2012) suffered from too many pixels that were masked out, reducing the effective number of reference images. They tended to oversubtract the target PSF and other point sources in the field. We therefore opted for a classical median subtraction, a trade-off between the quality of the PSF subtraction and source 
Table 4

$J$-band Observations for All Targets in the Sample

\begin{tabular}{lccccccr}
\hline \hline Name & Filter & Instrument & Obs. Date & Nexp & $\begin{array}{c}\text { Exposition Time } \\
(\mathrm{s})\end{array}$ & $\begin{array}{c}\text { FWHM } \\
\left({ }^{\prime \prime}\right)\end{array}$ & $\begin{array}{c}\text { Catalog } \\
\text { Conditions }\end{array}$ \\
\hline HIP 490 & J_G0802 & F2 & 2014 Jul 25 & 9 & 612 & 0.72 & median \\
HIP 560 & J_G0802 & F2 & 2014 Jul 25 & 9 & 612 & photometric \\
HIP 1113 & J_G0802 & F2 & 2016 Oct 10 & 14 & 630 & 2MASS & photometric \\
HIP 1134 & J & WIRCam & 2016 Aug 18 & 16 & 960 & 2MASS & photometric \\
HIP 1481 & J_G0802 & F2 & 2014 Jul 25 & 9 & 612 & 2MASS & patchy clouds \\
& & & & & & patchy clouds \\
\hline
\end{tabular}

(This table is available in its entirety in machine-readable form.)

Table 5

$z_{a b}^{\prime}$-band Observations for all Targets in the Sample

\begin{tabular}{|c|c|c|c|c|c|c|c|c|}
\hline Name & Filter & Instrument & Obs. Date & Nexp & $\begin{array}{c}\text { Exposition Time } \\
\text { (s) }\end{array}$ & $\begin{array}{c}\text { FWHM } \\
\left({ }^{\prime \prime}\right)\end{array}$ & Catalog & Conditions \\
\hline HIP 490 & z_G0328 & GMOS-S & 2014 Sep 09 & 8 & 1200 & 5.57 & skymapper & $\overline{\text { patchy clouds }}$ \\
\hline HIP 560 & z_G0328 & GMOS-S & 2014 Oct 10 & 8 & 1200 & 0.93 & panstarrs & patchy clouds \\
\hline HIP 1113 & z_G0328 & GMOS-S & 2015 Sep 02 & 8 & 520 & 0.76 & skymapper & patchy clouds \\
\hline HIP 1134 & z.MP9901 & MegaCam & 2016 Sep 07 & 4 & 1244 & 3.20 & skymapper & photometric \\
\hline HIP 1481 & z_G0328 & GMOS-S & 2014 Jul 20 & 9 & 1350 & 0.89 & median & patchy clouds \\
\hline
\end{tabular}

(This table is available in its entirety in machine-readable form.)

preservation. Following this strategy, the processed image under consideration was excluded from the library of references, the median of which was then taken as the reference PSF for subtraction of this image. The position of the star was estimated by fitting a Moffat function and the reference PSF was shifted to this position. The reference PSF was normalized to the target brightness within the same aperture and subtracted from the image. This three-step process was repeated for any low-contrast $(<1)$ visual companion of our target present in the field. For tight binaries or triple systems, the subtraction of all PSFs was done at once by iterating over the position and flux of each component in order to minimize the residuals in a box of width of 30 pixels. Saturated stars were processed like binaries to optimize the star registration and flux normalization. They still suffered from poorly subtracted wings and bright vertical stripes escaping from the core. Therefore, a new library was built from residual images of similarly saturated stars, following the same cleaning processes as for the original library. The median of this new library was used to subtract these residuals.

The images were finally high-pass filtered by subtracting a median filter of width 15 pixels.

\subsubsection{Archival Spitzer/MIPS $24 \mu m$ Data}

A search through the Spitzer archive revealed that 141 of our 177 targets were observed with MIPS at $24 \mu \mathrm{m}$ as part of surveys to find infrared excess indicative of a debris disk. A flux measurement (or upper limit) at such a longer wavelength can be useful to better constrain the SED of our candidate objects identified. Thus, for those 141 targets, we retrieved the MIPS data and built our own combined image using the Enhanced BCD images (EBCD), as they have a better flat fielding than the BCD image. A high-pass filter was then applied by subtracting double-pass median-filtered versions of the image of widths 32 and 12, respectively. We obtained the limiting flux in $\mathrm{Jy} / \mathrm{sr}$ by doing aperture photometry at several random positions over the whole image and then evaluating the robust standard deviation of the resulting flux distribution. We converted this limiting flux in $\mathrm{Jy} / \mathrm{sr}$ to magnitude and obtained a median limiting magnitude of 12.5 mag over all images.

\subsection{Photometric Calibration}

Our GEMINI observations were all acquired with a specification for observing conditions of up to $70 \%$ cloud cover, or patchy clouds. Under those conditions, a variation of up to $0.3 \mathrm{mag}$ can be expected. We assessed whether or not significant variations were present from the data themselves. For a sequence of observations of a given field, we calculated the standard deviation of the flux variations of the 20 brightest stars, as compared to a reference image from the sequence. If this variation was higher than $3 \%$, then we considered that the images of that target were not taken in photometric conditions ("patchy clouds" in Tables 4 and 5); otherwise we considered that the images were taken under photometric conditions ("phot" in Tables 4 and 5).

All of our CFHT images were taken in good conditions, with seeing around 0.6 for MegaCam and 1.1 for WIRCam, but we still checked the flux variations between images for a given target on a given night to make sure that the observations were acquired in photometric conditions.

Our final, stacked images in $z_{a b}^{\prime}$ and $J$ were calibrated in flux via comparison with the Sloan Digital Sky Survey catalog (SDSS DR9; Ahn et al. 2012) or the 2MASS All-Sky Catalog of Point Sources (Cutri et al. 2003), respectively. If SDSS data were not available for a given field, we used either PanSTARRS (Chambers \& Team 2018) $z_{p 1}$ data (available for 55 of our targets with decl. $>-30$ ) or SkyMapper (Wolf et al. 2018) $z^{\prime}$ data (available for 79 of our targets). The PanSTARRS filters $\left(g_{p 1}, r_{p 1}, i_{p 1}, z_{p 1}, y_{p 1}, w_{p 1}\right)$ are not the same as the SDSS filters, so we used the Tonry et al. (2012) color corrections to convert the Pan-STARSS magnitudes to SDSS magnitudes. For the $J$-band, if too few stars in our images were in the 2MASS PSC, we used deeper data from the VISTA Hemisphere Survey (VHS; McMahon et al. 2013) or archival 

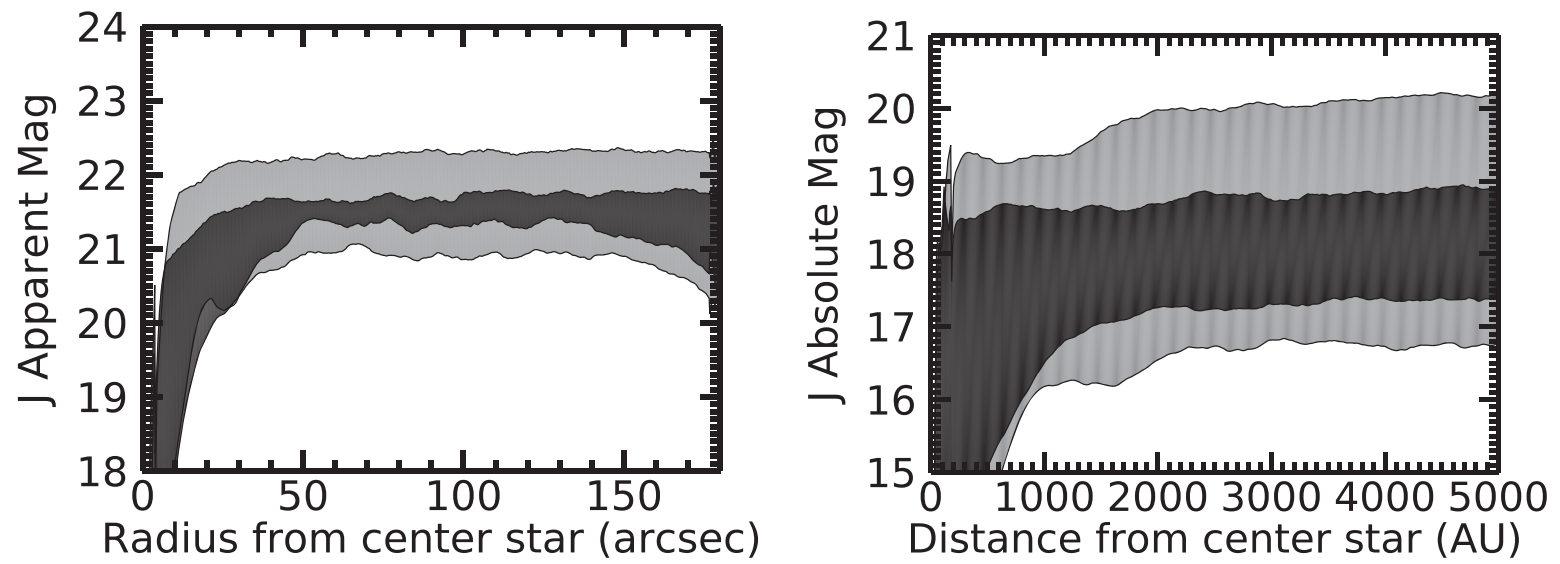

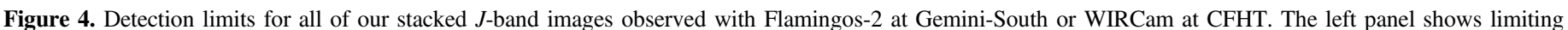

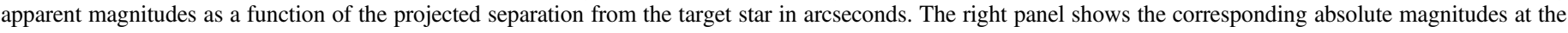

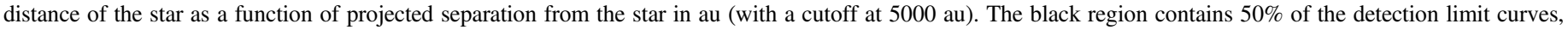
while $80 \%$ fall inside the gray region.

observations from the Observatoire du Mont Mégantic obtained using the Spectrographe infrarouge de Montréal (SIMON) (Albert 2006).

For a given image, the magnitude that produces one count per second on the detector, or the zero point, was calculated for each individual point source in common between our image and the catalog, based on the difference between the magnitude extracted from our image and the magnitude taken from the catalog. Next, the zero point of the image was taken to be the median of the individual zero points, and the error was computed by taking the standard deviation of those individual zero points divided by the square root of the number of sources.

When no catalog data were available for a given image, or when less than five objects with a magnitude measurement were available in the field of view, we used the median zero point for the given observing condition ("phot" or "patchy clouds"), instrument, and filter of the image. This occurred for 16 of our $J$-band images and 48 of our $z_{a b}^{\prime}$-band images. In the $J$-band, we obtained a zero point of $22.4 \pm 0.7$ and $22.1 \pm 0.9$ with Gemini/F2, and $22.5 \pm 0.8$ and $22.7 \pm 0.6$ with CFHT/ WIRCam, for photometric and non-photometric conditions respectively. In the $z$-band, we calculated a median zero point of $24.5 \pm 0.3$ and $24.6 \pm 0.5$ with CFHT/MegaCam, and $29.7 \pm 2$ and $28.9 \pm 2.3$ with Gemini/GMOSS, for photometric and non-photometric conditions respectively.

\subsection{Follow-up Observations}

Our search for planetary-mass objects revealed a number of candidates (see Section 3.2.3), which motivated us to obtain follow-up observations.

An astrometric follow-up was carried out between 2016 and 2017 in the J-band, with either CFHT/WIRCam or GeminiSouth/Flamingos2. Only $J$-band images were obtained, as it is in this band that the $\mathrm{S} / \mathrm{N}$ of the candidate is highest. We used the same observation parameters as for the first epoch observations. We obtained proper motions follow-up in the $J$-band for four candidate companions.

\section{Results}

The ground-based observations described earlier were designed to reach a limiting magnitude of $z=24$ mag at $3 \sigma$ and $J=21 \mathrm{mag}$ at $7 \sigma$. In practice, we achieved a median [AB] limiting magnitude in the $z$-band of $23.4 \pm 1.2 \mathrm{mag}$ with CFHT/MegaCam, and $23.7 \pm 1.2 \mathrm{mag}$ with Gemini/GMOS$\mathrm{S}$, at $3 \sigma$. In the $J$-band, we achieved a $7 \sigma$ median Vega limiting magnitude of $21.2 \pm 0.5 \mathrm{mag}$ with CFHT/WIRCam, and $21.0 \pm 0.8 \mathrm{mag}$ with Gemini/F2. For the Spitzer/IRAC observations, we reached a median magnitude limit of $18.5 \pm 0.9 \mathrm{mag}$ at [3.6] at $5 \sigma$, and $18.5 \pm 0.8 \mathrm{mag}$ at [4.5] at $3 \sigma$.

\subsection{Detection Limits}

The sensitivities to companions, in terms of limiting magnitudes, were evaluated for each $J$-band stacked image and [4.5] image as a function of the radial distance from the target star. For each radius from the central star, aperture photometry was performed by obtaining the flux inside 100 apertures of radius of 1 FWHM and a sky annulus between 4-6 FWHM. The limiting flux at each radius is the standard deviation of these 100 fluxes and it was then converted into magnitudes to get these $7 \sigma$ limiting magnitudes. These results are presented in Figure 4 for the $J$-band images and in Figure 5 for the [4.5] images. They show that the limiting magnitudes initially grow with increasing distance from the central star, and then reach a plateau. The black regions of the plots contain $50 \%$ of the detection limit curves, while $80 \%$ of the curves fall inside the gray region. For the $J$-band images, the plateau is reached at $\sim 30^{\prime \prime}$ at a magnitude of $J \sim 21.5 \mathrm{mag}$ for $50 \%$ of our target stars (the black region). The curves are truncated at about $180^{\prime \prime}$, which corresponds to the limit of the field of view of the Flamingos-2 images. The plateau is reached at a projected physical separation of 1000 au for an average star of our sample. For our [4.5] images, the plateau at magnitude $\sim 18.5$ is reached at a radius of $\sim 50^{\prime \prime}$ for $50 \%$ of the stars of the sample (the black region). We used the same cut-off as the $J$-band images. Tables 6 and 7 present, respectively for the $J$-band and [4.5] images, the $7 \sigma$ detection limits for each target over the plateau, along with the minimum and maximum separations (in arcsec and au) where these limits are valid (defined as the range for which the detection limit is, at most, one magnitude brighter than the plateau value given, to accommodate for small fluctuations with separations).

The limiting magnitudes can be converted to limiting masses using evolutionary models at the ages of our targets (which range between 10 and $150 \mathrm{Myr}$ ). We used the COND models 

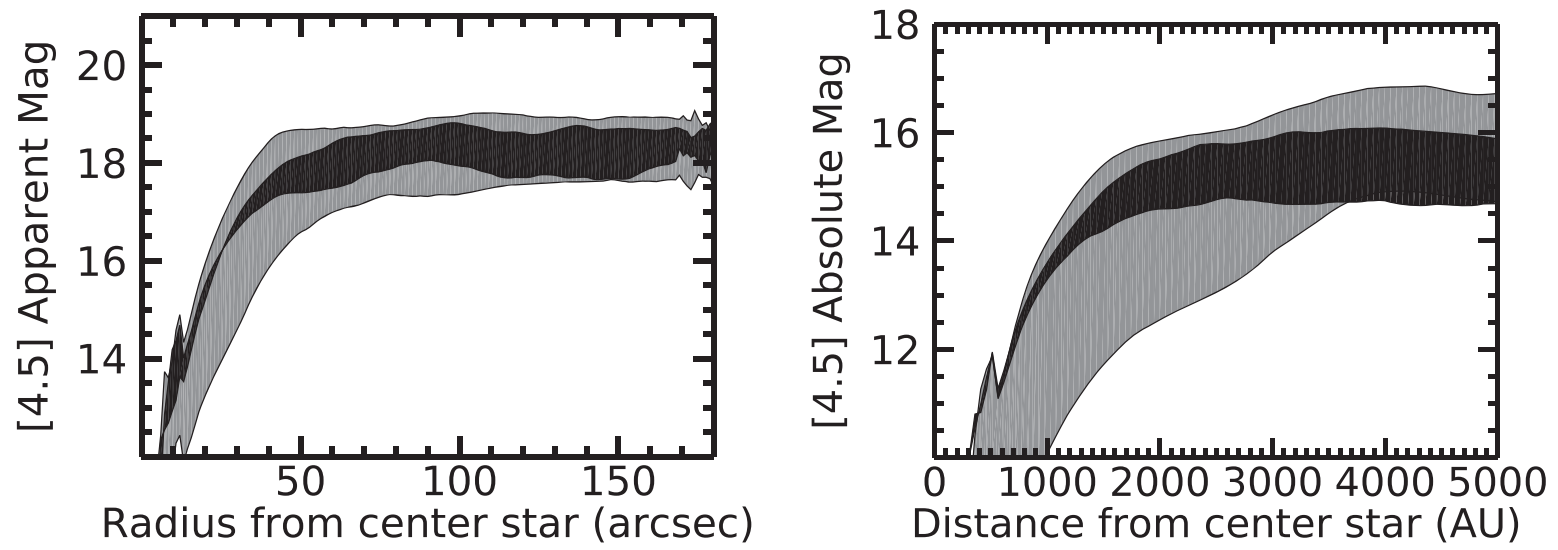

Figure 5. Same as 4 for the Spitzer/IRAC observations.

Table 6

$7 \sigma$ Detection Limits in the $J$-Band

\begin{tabular}{lccccccc}
\hline \hline Name & $\begin{array}{c}a_{\min } \\
(')\end{array}$ & $\begin{array}{c}a_{\max } \\
\left({ }^{\prime}\right)\end{array}$ & $\begin{array}{c}a_{\min } \\
(\mathrm{au})\end{array}$ & $\begin{array}{c}a_{\min } \\
(\mathrm{au})\end{array}$ & $J$ & $\begin{array}{c}M_{J} \\
\left({ }^{\prime}\right)\end{array}$ & $\begin{array}{c}\text { Mass Limit } \\
\left(M_{\text {Jup }}\right)\end{array}$ \\
\hline HIP 490 & 32 & 180 & 1241 & 7096 & 22.1 & 19.1 & 1.0 \\
HIP 560 & 46 & 180 & 1808 & 7096 & 20.7 & 17.7 & 1.7 \\
HIP 1113 & 5 & 180 & 232 & 8001 & 21.0 & 17.7 & 1.7 \\
HIP 1134 & 19 & 630 & 877 & 29717 & 21.8 & 18.5 & 1.3 \\
HIP 1481 & 27 & 180 & 1137 & 7486 & 20.6 & 17.5 & 1.8 \\
\hline
\end{tabular}

(This table is available in its entirety in machine-readable form.)

Table 7

$7 \sigma$ Detection Limits in the [4.5] Band

\begin{tabular}{lccccccc}
\hline \hline Name & $\begin{array}{c}a_{\min } \\
\left({ }^{\prime}\right)\end{array}$ & $\begin{array}{c}a_{\max } \\
\left({ }^{\prime}\right)\end{array}$ & $\begin{array}{c}a_{\min } \\
(\mathrm{au})\end{array}$ & $\begin{array}{c}a_{\min } \\
(\mathrm{au})\end{array}$ & {$[4.5]$} & $\begin{array}{c}M_{[4.5]} \\
\left({ }^{\prime}\right)\end{array}$ & $\begin{array}{c}\text { Mass Limit } \\
\left(M_{\text {Jup }}\right)\end{array}$ \\
\hline HIP 490 & 28 & 479 & 1087 & 18858 & 18.7 & 15.7 & 0.3 \\
HIP 560 & 25 & 479 & 993 & 18858 & 18.4 & 15.4 & 0.4 \\
HIP 1113 & 28 & 479 & 1226 & 21261 & 18.8 & 15.6 & 0.3 \\
HIP 1134 & 24 & 479 & 1132 & 22574 & 18.2 & 14.8 & 0.7 \\
HIP 1481 & 25 & 479 & 1047 & 19892 & 18.5 & 15.4 & 0.4 \\
\hline
\end{tabular}

(This table is available in its entirety in machine-readable form.)

from Baraffe et al. (2003) to infer the masses. These models assume a hot start, as described by Bowler (2016), which corresponds to idealized initial conditions and an arbitrarily large initial radius. This model is thus optimistic, as it represents more luminous planets than do cold start models. The mass limit reached over the sensitivity plateau for each target is indicated in Tables 6 and 7.

\subsection{Candidate Search}

We searched for and identified candidates in our imaging based on their $z_{a b}^{\prime}-J$ and [3.6]-[4.5] colors. We started by identifying all point sources in the $J$-band images using the IDL find procedure (from Astrolib) and then fitted a 2D Gaussian function to each of them to get a more precise position. At this step, sources with an elongated PSF were rejected, as a first attempt to exclude extra galactic contaminants. We also rejected sources too close to the edge of the field (for F2 or GMOS-S) and sources that were saturated in either band. We used coordinates measured in our $J$-band images to identify

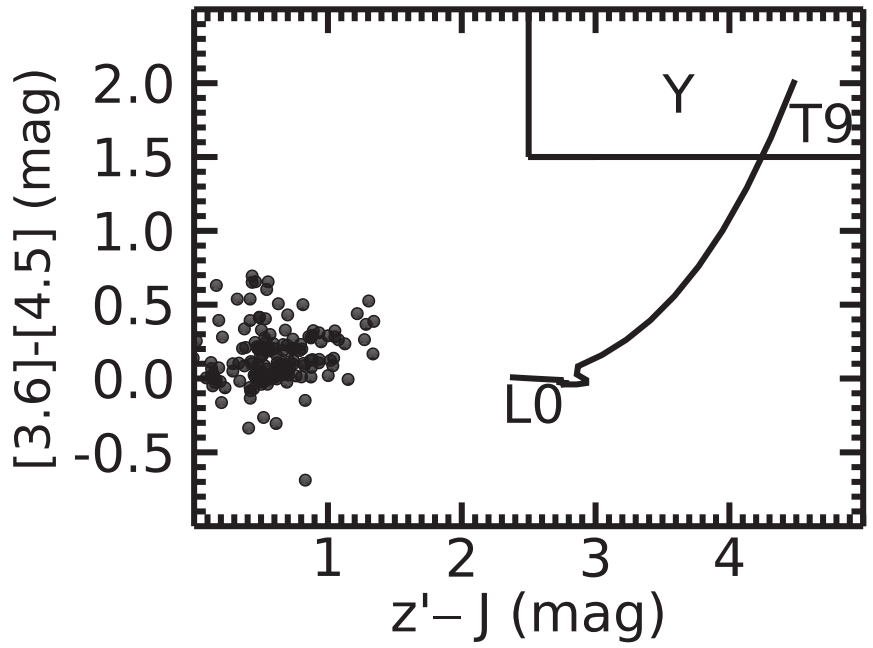

Figure 6. Color-color diagram for HIP 26453, a known member of Columba. The dots represent all sources detected in our $J$-band imaging, and without detection in Gaia, within a radius of 5000 au from the target star. The solid line shows the expected color sequence for spectral types L to T from Dupuy \& Liu (2012). The box represents the expected colors for early Y dwarfs. No candidates were detected in this field.

sources in the $z_{a b}^{\prime}$-band images. In both bands, we used aperture photometry with a radius of 1 FWHM and a sky sampling annulus extending between 2-3 FHWM to retrieve the instrumental flux of each source. We kept only point sources detected at $7 \sigma$ in $J, 5 \sigma$ in [4.5] and $3 \sigma$ in [3.6]. At a distance of $>20 \mathrm{pc}$, which is the case for $90 \%$ of the stars in our sample, a radius of 5000 au fits in the field of view of the Spitzer/IRAC images. For that reason, we searched for candidates only inside a projected separation of 5000 au from the target stars.

We found the center of the target star by fitting a 2D Gaussian to the PSF, for stars that were not saturated. However, most of our targets were saturated in our $J$-band images. Thus, we used the Gaia DR1 DR1 catalog (Gaia Collaboration et al. 2016) to find an approximate position for the star. We then used the known proper motion of the star to compute its position at the time the image was obtained. If Gaia data were not available, we fitted a 2D Gaussian to the PSF, where all of the saturated pixels were given the maximum value possible for a pixel. For the IRAC images, the center of the stars was obtained during the PSF removal process. 


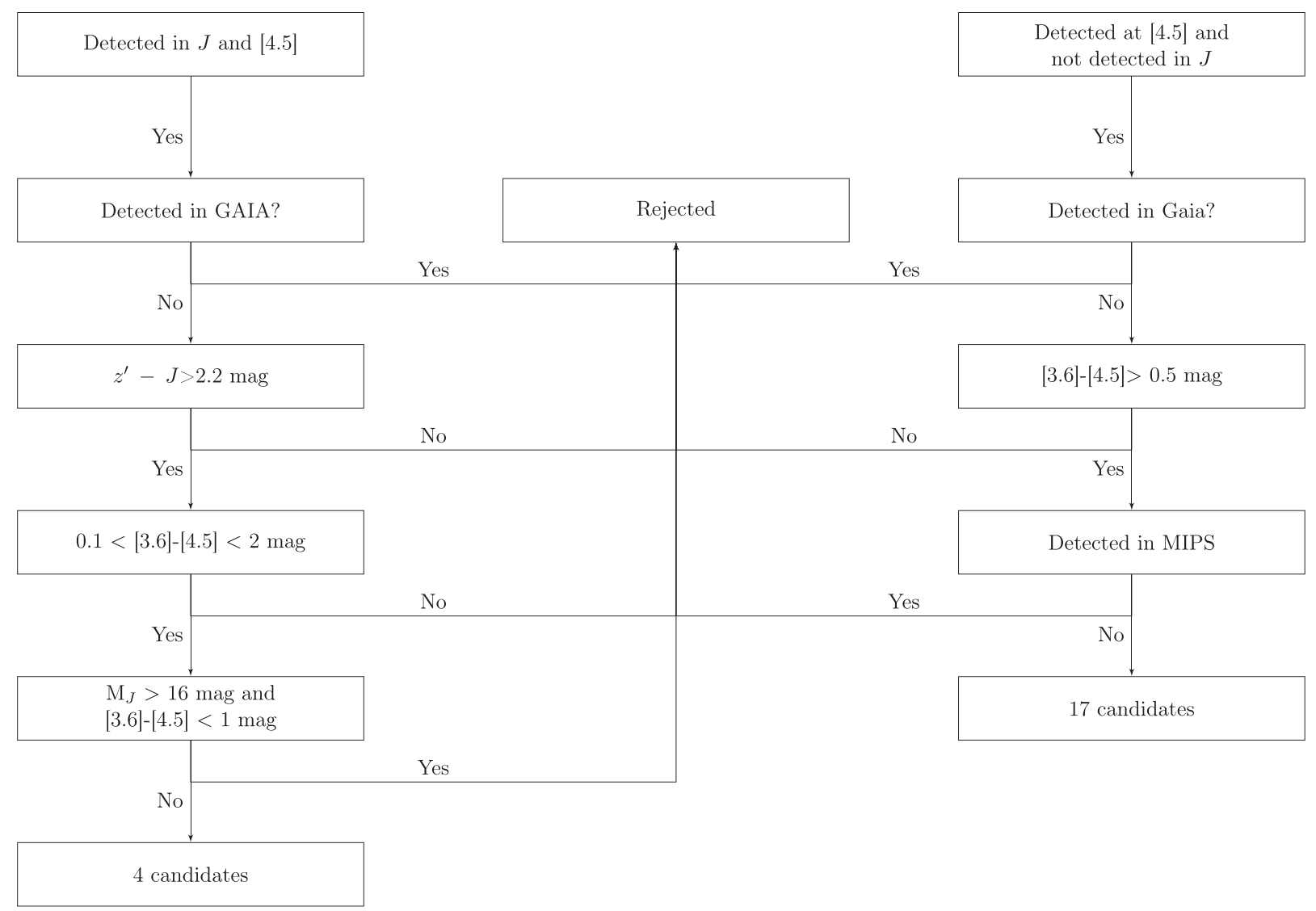

Figure 7. Flowchart of the candidate selection. Candidates detected in the $J$-band are on the left, and the IRAC-only candidates are on the right.

\subsubsection{Colors}

For the ages of our target stars, 10-150 Myr, the transition between brown dwarfs and planets happens between L1/L2 and L5/L6, based on AMES-Cond models (Baraffe et al. 2003). As mentioned above (and see Figure 2), early-type $\mathrm{L}$ dwarfs have a $z_{a b}^{\prime}-J$ color $\gtrsim 2.5 \mathrm{mag}$. Considering our errors on magnitudes and zero points, we selected only sources with $z_{a b}^{\prime}-J>2.2$. Per the above discussion, this same color cut is also sensitive to T and Y dwarfs, which can be identified either through detection in both bands or as $z_{a b}^{\prime}$ dropouts (in the cases without detection in $z_{a b}^{\prime}$, we get only a lower limit on the $z_{a b}^{\prime}-J$ color). Thus, at this stage, we kept all sources with $z_{a b}^{\prime}-J>2.2 \mathrm{mag}$, including all the $z_{a b}^{\prime}$ dropouts.

As a second step, we removed any source that has a counterpart in the Gaia DR1 catalog (Gaia Collaboration et al. 2016). Gaia can detect objects with magnitude as low as $G=20$. Based on the relationship between $G-J$ and the spectral type of L dwarfs from Smart et al. (2017), using the expected $J$ magnitude of L dwarfs from Faherty et al. (2016), and assuming a distance of $42 \mathrm{pc}$ (the median distance of our sample), the cut in Gaia magnitudes rejects objects earlier than $\sim \mathrm{L} 2$.

Next, we compared the $z_{a b}^{\prime}-J$ colors and [3.6]-[4.5] colors of our candidates to typical colors of ultracool field dwarfs (Dupuy \& Liu 2012); see Figure 6. This figure shows all point sources in a radius of $5000 \mathrm{au}$ in the $J$-band image for an average target of the sample for which there was no candidate detected. The solid black line represents the expected colors for L to T dwarfs according to Dupuy \& Liu (2012). We kept as candidates only the detections with [3.6]-[4.5] $\sim 0.1$ to
$2 \mathrm{mag}$, as this is the expected interval for T dwarf's colors. We also kept as candidates sources with $M_{J}<16$ mag and [3.6][4.5] $<1 \mathrm{mag}$ or $M_{J}>16 \mathrm{mag}$ and [3.6]-[4.5] $>1 \mathrm{mag}$. Figure 7, right, presents the flowchart of the candidate selection for the candidates detected in the $J$-band.

In some cases, a source was detected at $5 \sigma$ in our IRAC data but we found no counterpart in our $J$ or $z_{a b}^{\prime}$ imaging, respectively at 7 and $3 \sigma$. Unambiguous IRAC-only detection of planets is possible only if [3.6]-[4.5] $>2$, which corresponds to our detection limits of $\sim 21$ in the $J$-band, or $M_{J} \sim 18$ (T8.5) at $50 \mathrm{pc}$ according to AMES-Cond models. However, most of our IRAC-only detection had $0.5<[3.6]-$ $[4.5]<2$. As the color in those bands for young 2 Mjup objects is rather uncertain, we decided to follow-up these sources anyway. Thus, from the IRAC-only detection, we selected only sources with [3.6]-[4.5] $>0.5$ and no Gaia detection. In addition, as the absolute magnitudes of young planetary mass objects analog to T dwarfs are not well-known, we kept only sources with a [4.5] absolute magnitude within $0.75 \mathrm{mag}$ from the typical values of field $\mathrm{T}$ dwarfs; see Figure 8. This method uncovered 79 candidates with the expected colors of T dwarfs. Figure 7, on the right, presents the flowchart of the candidate selection for the candidates not detected in the $J$-band.

The color criteria above yielded typically a few candidates per field. However, most were easily discarded by looking at either the stacked images or the individual frames: some had an elongated PSF that escaped our automatic cut, some fell out of the detector in one or more frames of the dither pattern biasing their photometry, some were due to a persistent signal from a bright star that was on the same part of the detector in a 


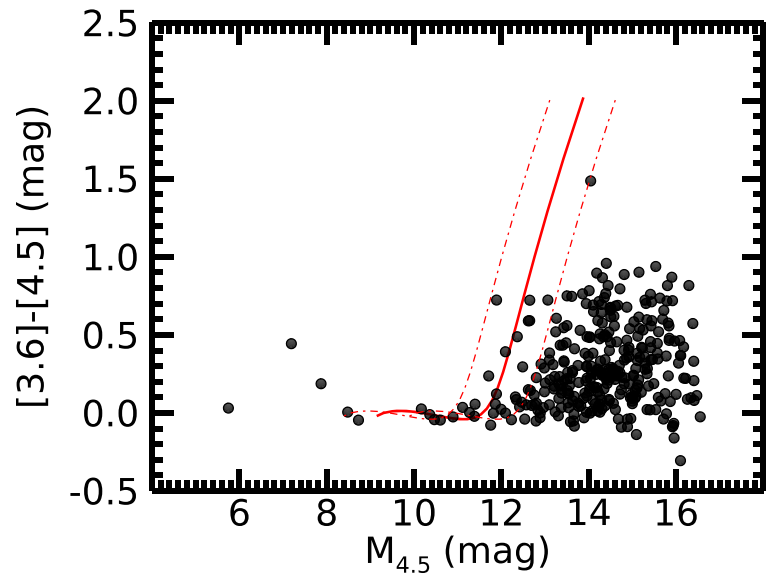

Figure 8. [3.6]-[4.5] color of sources detected in our Spizter imaging of HIP 11152 vs. their [4.5] absolute magnitude at the distance of the target star. The solid red line corresponds to the colors of M6 to T9 dwarfs from Dupuy \& Liu (2012). The dotted lines on either sides represent a spread of 0.75 magnitude. The dots are all the point sources presents in a sphere of 5000 au around the central star for which there is no detection in the optical. One point source has colors consistent with a late- $\mathrm{T}$ dwarf at the right absolute magnitude. This point source is not detected in the $z_{a b}^{\prime}$ or $J$ images. While it is expected for a planetary-mass companion to be undetected in $z_{a b}^{\prime}$, it should have been detected in $J$ images, given our detection limits. It is thus likely that the candidate is in fact an extragalactic contaminant.

previous frame (for the WIRCam images), and some fell over the spider diffraction spikes of the host star. After these initial verifications, our search yielded four candidates with $J$-band detection and 48 candidates with IRAC-only detections.

\subsubsection{Cross-match with the 2MASS Catalog}

The detection method described earlier is not sensitive to companions with spectral types earlier than an early-L dwarf. Instead, the latest $\mathrm{M}$ to early L-type dwarf companions can be identified through a search for common proper motion based on a comparison of our $J$-band images with 2MASS images, given the $\sim 15$ year baseline between them. We performed such a proper motion comparison for all sources with $J<16.5 \mathrm{mag}$.

This search identified one candidate with a proper motion consistent with a target star: TWA30B, an M4V dwarf companion of TWA30 (which is an M5 dwarf member of the TW Hydrae association) at a separation of 3400 au. It was discovered previously by Looper et al. (2010).

\subsubsection{Follow-up of Candidates}

The follow-up of our candidate companions includes three different types of observations. First of all, IRAC-only detections were studied in greater detail by using MIPS data. A photometric follow-up was obtained to try to identify puzzling objects with very red [3.6]-[4.5] colors and no detection in the $z_{a b}^{\prime}$ and $J$-bands. Last, a proper motion followup was obtained for all candidates detected in $J$ that survived the color cuts and verifications.

Our search for candidates in the Spitzer/IRAC images yielded 48 candidates with [3.6]-[4.5] $>0.5$ and no detection in $z^{\prime}$ or $J$. Figure 8 shows all the point sources detected in [3.6] and [4.5] in a given field and for which no visible counterpart was found (from the Gaia DR1 catalog). Faint, red objects like this certainly constitute interesting planetary-mass candidates, as it is expected for such objects to be $z^{\prime}$ dropouts. However, given our limits, it is unexpected for them to be unseen in $J$.

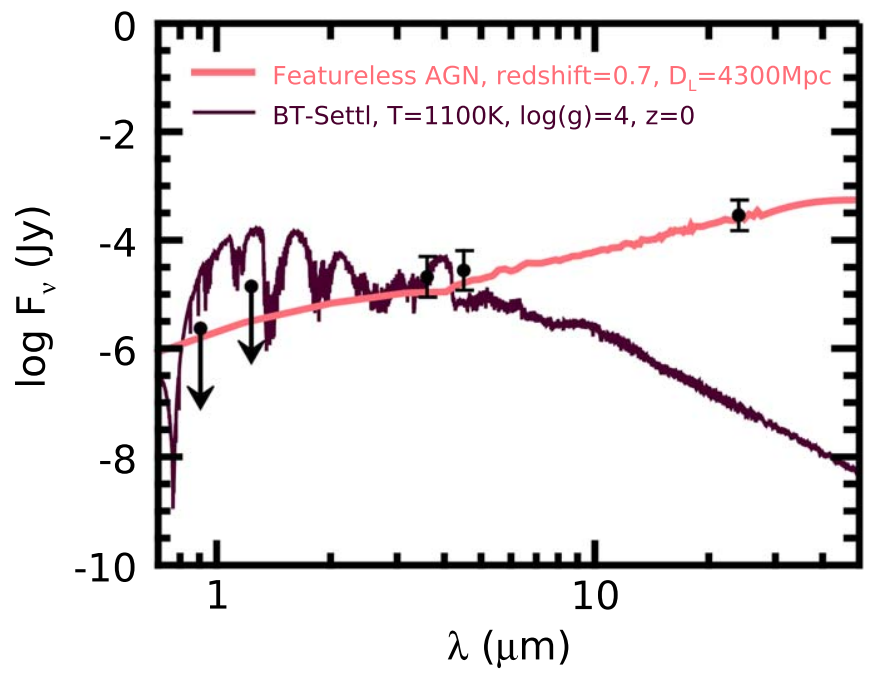

Figure 9. Photometric data for one candidate that has a large [3.6]-[4.5] color but no detection in $z_{a b}^{\prime}$ and $J$. The data are compared to the model spectrum of an object with a $T_{\text {eff }}=1100 \mathrm{~K}, \log g=4$, and $z=0$ from BT-Settl (purple), as well as to the spectrum of a featureless AGN with a redshift of 0.7 and a $D_{L}=4300 \mathrm{Mpc}$ (magenta, from Kirkpatrick et al. 2012a). We see that the detection at $24 \mu \mathrm{m}$ makes it very easy to untangle between a mid-T dwarf and an AGN.

Other astrophysical sources that may have similar photometric properties include galaxies and active galactic nuclei (AGNs).

Figure 9 shows the expected SED of a low-mass object with an effective temperature of $1100 \mathrm{~K}$ compared to the SED of a featureless AGN. As the figure illustrates, it is difficult to untangle AGNs from planetary candidates using [3.6] and [4.5] photometry alone, but photometry at $24 \mu \mathrm{m}$ is a very good discriminator. We used the MIPS $24 \mu \mathrm{m}$ images mentioned above, reaching a limiting magnitude of 12.5 at $1 \sigma$ in that band for most targets, to see if our candidates were detected at that wavelength, which would be incompatible with a planetarymass object. This enabled us to reject 31 of our remaining IRAC-only candidates and to identify them as extragalactic contaminants. We checked archives to see whether those MIPS detection are associated with X-ray or radio emission, but none of them are already known to be an AGN.

After this cut, 17 IRAC-only candidates remain. Figure 10 shows the colors and magnitudes of the candidates compared to different models as well as to photometric data from known young $\mathrm{T}$ dwarfs. Of those 17 candidates, four were observed by MIPS but not detected. These candidates have [3.6]$[4.5]=0.7-0.9$ mag and [4.5] magnitudes between between $15.8 \mathrm{mag}$ and $17.5 \mathrm{mag}$. Using only their IRAC color and assuming that they are T dwarfs and that the BT-Settl/AMESCond model are valid, one would expect them to have $M_{J} \sim$ 15 mag, which would have been detected by our survey. As they show no detection in our $J$-band imaging, we rejected those four candidates. The last 13 candidates were not observed by MIPS. Those candidates have [3.6]-[4.5] colors between $0.6 \mathrm{mag}$ and $1.3 \mathrm{mag}$ and [4.5] magnitudes between 15.2 and 16.9 mag. Using the same thought process as for the candidates not seen in MIPS, we see that those candidates also should have been seen in the $J$-band, but they were not. We thus reject the last 13 candidates, so no IRAC-only candidate remain. However, we decided to list those 17 rejected candidates in the interest of completeness, as we cannot identify the nature of the candidates at this stage, and because models might not reproduce accurately the colors of young late- $\mathrm{T}$ to early- $\mathrm{Y}$ 

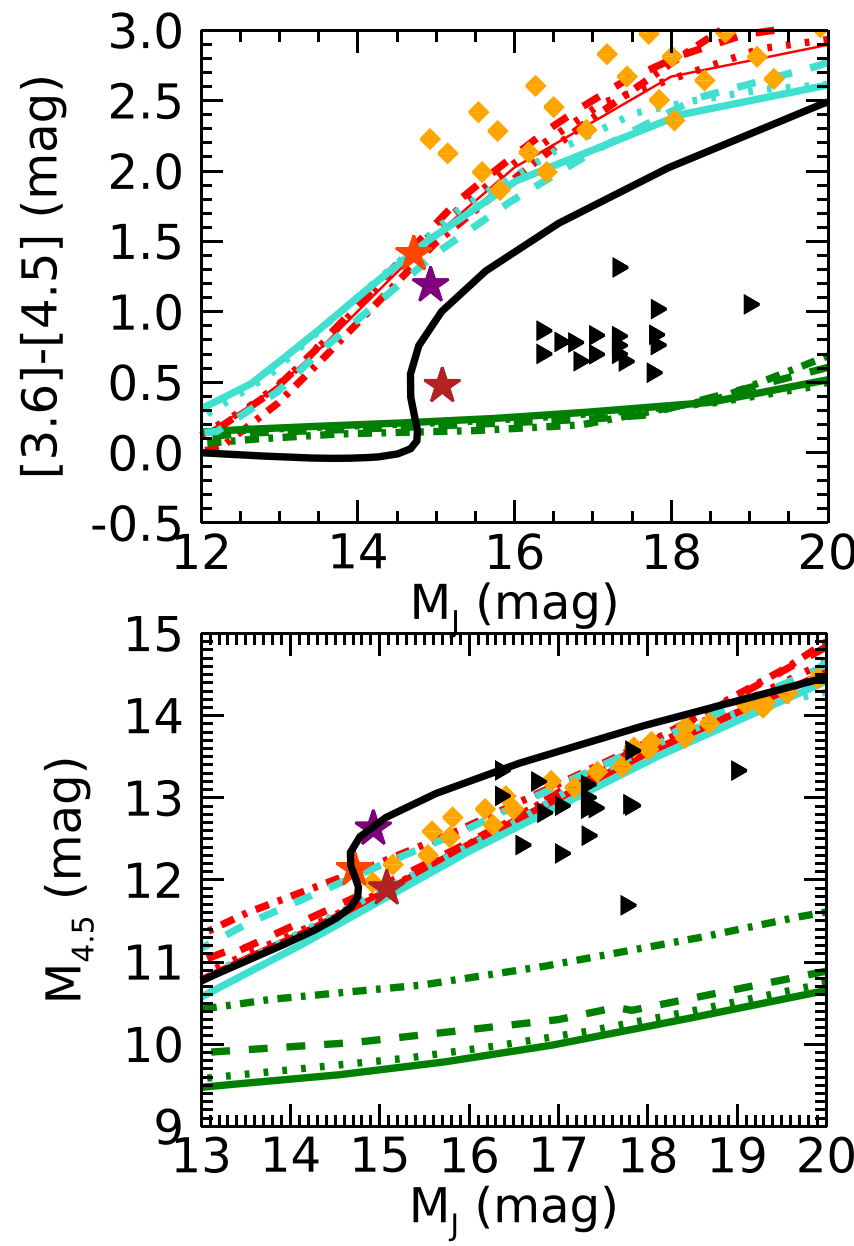
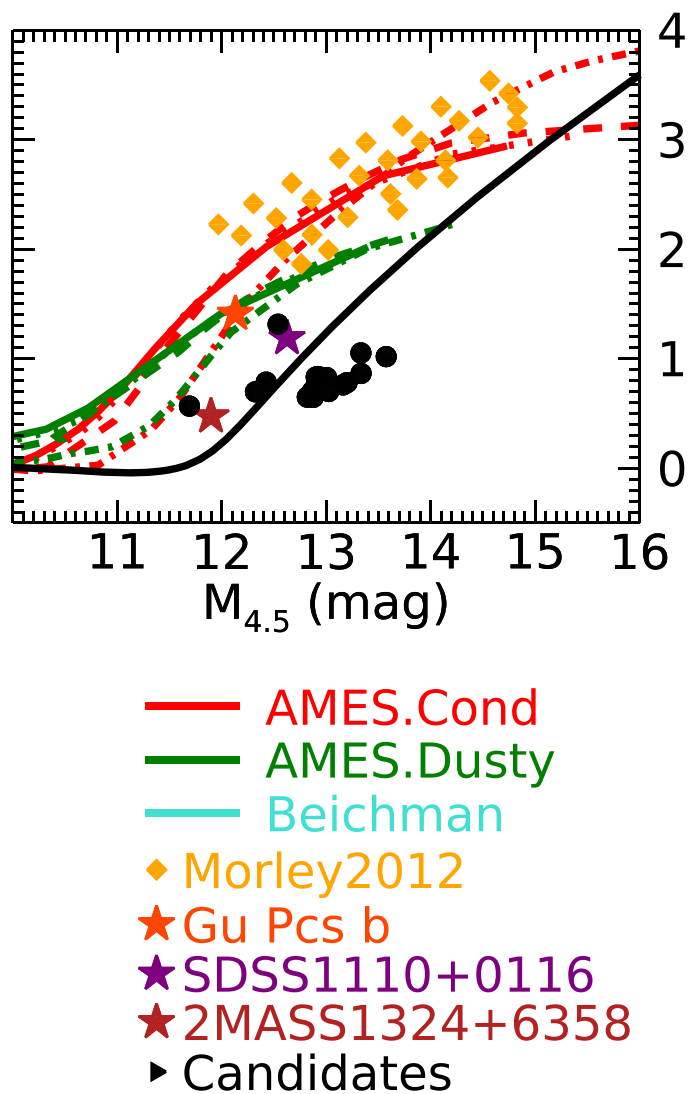

Figure 10. Colors of our 17 Spitzer/IRAC-only candidates remaining after the MIPS detection cut (triangles, upper limits in J-band). The [3.6]-[4.5] colors vs. absolute $J$ magnitude are shown on the upper left, while [3.6]-[4.5] colors vs. absolute [4.5] magnitudes are displayed on the upper right. The lower left shows absolute [4.5] magnitudes vs. absolute $J$ magnitudes. Colors for M6 to T9 dwarfs from Dupuy \& Liu (2012) are shown with a black line. The red curves represent the AMES-Cond models (Baraffe et al. 2003) at 10, 20, 120, and 5000 Myr, using respectively the solid, dotted, dashed, and dashed-dotted line. Also shown are models from Beichman et al. (2014) in cyan, Mordasini et al. (2012) in yellow, and AMES-Dusty in green. Photometric data for three young T dwarfs are also shown by an orange star for Gu Psc b (Naud et al. 2014), a purple star for SDSS1110+0116 (Gagné et al. 2015a), and a red orange star for 2MASS1324+6358 (Gagné et al. 2018a). While the candidate companions have [3.6]-[4.5] colors vs. [4.5] similar to those of the young T dwarfs, they are too faint in the $J$-band to be considered planetary objects.

Table 8

Properties of the Candidates without MIPS Data or Detection

\begin{tabular}{|c|c|c|c|c|c|c|c|c|}
\hline $\begin{array}{l}\text { R.A. } \\
\text { (J2000.0) }\end{array}$ & $\begin{array}{c}\text { Decl. } \\
\text { (J2000.0) }\end{array}$ & $\begin{array}{l}\text { Host Star } \\
\text { (mag) }\end{array}$ & $\begin{array}{c}z_{a b}^{\prime} \\
(\mathrm{mag})\end{array}$ & $\begin{array}{c}J^{\prime} \\
(\mathrm{mag})\end{array}$ & $\begin{array}{l}{[3.6]} \\
(\mathrm{mag})\end{array}$ & $\begin{array}{c}{[4.5]} \\
\left({ }^{\prime}\right)\end{array}$ & $\begin{array}{l}\text { Separation } \\
\text { (pc) }\end{array}$ & Distance \\
\hline 02:46:00.708 & $05: 35: 00.82$ & HIP 12925 & $>23.83$ & $>21.48$ & $16.68 \pm 0.08$ & $16.76 \pm 0.08$ & $67.5130 \pm 0.019$ & $54.3 \pm 3.0$ \\
\hline 02:50:00.567 & $27: 16: 00.52$ & HIP 13209 & $>22.73$ & $>20.93$ & $15.59 \pm 0.07$ & $15.62 \pm 0.07$ & $69.7032 \pm 0.021$ & $50.7 \pm 0.4$ \\
\hline 04:48:00.751 & $-80: 46: 00.34$ & HIP 22295 & $>24.75$ & $>21.71$ & $18.42 \pm 0.08$ & $18.11 \pm 0.08$ & $107.158 \pm 0.188$ & $61.0 \pm 1.8$ \\
\hline 05:01:00.177 & $-20: 03: 00.08$ & HIP 23362 & $>24.04$ & $>20.94$ & $18.62 \pm 0.08$ & $17.83 \pm 0.08$ & $57.8078 \pm 0.017$ & $60.6 \pm 2.1$ \\
\hline 05:01:00.270 & $-20: 01: 00.43$ & HIP 23362 & $>24.04$ & $>20.94$ & $17.77 \pm 0.08$ & $17.32 \pm 0.08$ & $80.2475 \pm 0.022$ & $60.6 \pm 2.1$ \\
\hline 05:20:00.536 & $-39: 45: 00.03$ & HIP 24947 & $>22.15$ & $>20.00$ & $18.02 \pm 0.08$ & $17.71 \pm 0.08$ & $97.8883 \pm 0.035$ & $48.3 \pm 0.9$ \\
\hline 06:00:00.277 & $-44: 53: 00.56$ & HIP 28474 & $>24.82$ & $>20.92$ & $18.36 \pm 0.05$ & $18.07 \pm 0.05$ & $75.5571 \pm 0.029$ & $52.5 \pm 1.6$ \\
\hline 06:00:00.613 & $-44: 52: 00.49$ & HIP 28474 & $>24.82$ & $>20.92$ & $17.78 \pm 0.05$ & $17.83 \pm 0.05$ & $80.8067 \pm 0.024$ & $52.5 \pm 1.6$ \\
\hline 06:00:00.859 & $-44: 53: 00.07$ & HIP 28474 & $>24.82$ & $>20.92$ & $17.80 \pm 0.25$ & $17.50 \pm 0.25$ & $83.5713 \pm 0.032$ & $52.5 \pm 1.6$ \\
\hline $06: 46: 00.315$ & $-83: 59: 00.83$ & HIP 32435 & $>25.06$ & $>20.50$ & $19.38 \pm 0.08$ & $18.65 \pm 0.08$ & $65.1345 \pm 0.164$ & $56.0 \pm 1.1$ \\
\hline 07:00:00.902 & $-79: 40: 00.69$ & HIP 33737 & $>24.87$ & $>20.67$ & $18.00 \pm 0.08$ & $18.04 \pm 0.08$ & $70.2442 \pm 0.063$ & $58.8 \pm 3.0$ \\
\hline 09:36:00.173 & $-78: 19: 00.80$ & HIP 47135 & $>24.45$ & $>20.51$ & $17.61 \pm 0.25$ & $17.54 \pm 0.25$ & $63.8135 \pm 0.035$ & $67.9 \pm 2.7$ \\
\hline 09:36:00.649 & $-78: 19: 00.76$ & HIP 47135 & $>24.45$ & $>20.51$ & $19.02 \pm 0.25$ & $18.64 \pm 0.25$ & $73.1604 \pm 0.039$ & $67.9 \pm 2.7$ \\
\hline 11:39:00.275 & $-32: 00: 00.12$ & TWA26 & $>24.43$ & $>20.93$ & $17.50 \pm 0.08$ & $17.38 \pm 0.08$ & $78.5368 \pm 0.024$ & $41.9 \pm 4.5$ \\
\hline 23:05:00.214 & 63:58:00.78 & HIP 114066 & $>22.46$ & $>20.94$ & $18.58 \pm 0.07$ & $17.62 \pm 0.07$ & $192.299 \pm 0.072$ & $24.5 \pm 0.9$ \\
\hline 23:11:00.882 & $-45: 08: 00.82$ & HIP 114530 & $>23.99$ & $>21.29$ & $16.33 \pm 0.05$ & $16.29 \pm 0.05$ & $78.7520 \pm 0.030$ & $50.7 \pm 2.8$ \\
\hline 23:40:00.795 & 44:18:00.89 & HIP 116805 & $>24.28$ & $>20.89$ & $17.06 \pm 0.25$ & $16.96 \pm 0.25$ & $87.3615 \pm 0.024$ & $51.6 \pm 0.5$ \\
\hline
\end{tabular}


Table 9

Properties of the Candidates Identified Through Color Cuts

\begin{tabular}{|c|c|c|c|c|c|c|c|c|c|c|}
\hline $\begin{array}{l}\text { Host Star } \\
\text { (J2000.0) }\end{array}$ & $\begin{array}{c}\text { R.A. } \\
\text { (J2000.0) }\end{array}$ & Decl. & $\begin{array}{l}M_{z_{a b}^{\prime}} \\
\text { (mag) }\end{array}$ & $\begin{array}{c}M_{J} \\
(\mathrm{mag})\end{array}$ & $\begin{array}{l}M_{[3.6]} \\
(\mathrm{mag})\end{array}$ & $\begin{array}{l}M_{[4.5]} \\
\text { (mag) }\end{array}$ & $\begin{array}{l}\text { Separation } \\
\quad \text { (au) }\end{array}$ & $\begin{array}{c}\mu_{\alpha} \cos \delta \\
\left(\mathrm{mas} \mathrm{yr}^{-1}\right)\end{array}$ & $\begin{array}{c}\mu_{\delta} \\
\left(\operatorname{mas~yr}^{-1}\right)\end{array}$ & $\begin{array}{c}\text { Rejected at } \\
\text { (sigma) }\end{array}$ \\
\hline HIP $14913 \mathrm{~A}$ & 48.085635 & -44.426938 & $18.26 \pm 0.13$ & $15.98 \pm 0.06$ & $13.82 \pm 0.10$ & $13.31 \pm 0.09$ & $5559 \pm 101$ & $-46.71 \pm 26.00$ & $16.75 \pm 30.2700$ & 3 \\
\hline HIP 29964 & 94.595099 & -72.054682 & $18.35 \pm 1.70$ & $16.88 \pm 0.02$ & $14.46 \pm 0.07$ & $13.46 \pm 0.05$ & $1670 \pm 116$ & $27.52 \pm 17.48$ & $12.81 \pm 22.5900$ & 3 \\
\hline HIP 79881 & 244.61165 & -28.608996 & $18.35 \pm 1.60$ & $16.36 \pm 0.09$ & $14.05 \pm 0.06$ & $13.31 \pm 0.05$ & $6365 \pm 442$ & $3.73 \pm 9.76$ & $-6.13 \pm 9.53000$ & 8 \\
\hline HD 152555 & 253.51768 & -4.3371192 & $18.83 \pm 0.07$ & $16.46 \pm 0.12$ & $14.08 \pm 0.12$ & $13.65 \pm 0.11$ & $4895 \pm 89$ & $-10.10 \pm 13.25$ & $-12.86 \pm 17.6900$ & 5 \\
\hline
\end{tabular}




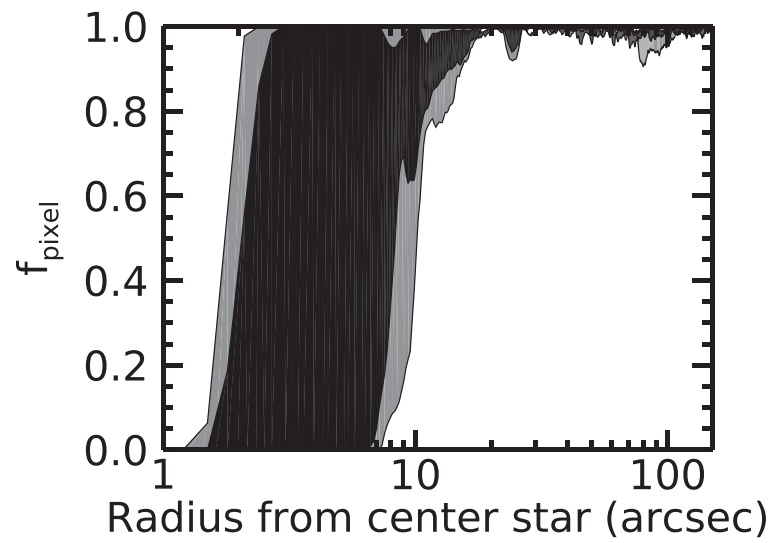

Figure 11. Fraction of clean pixels where a companion could be detected as a function of the separation from the target star in the $J$-band images. Fifty percent of the stars have a fraction of pixel that is included in the black area, while the gray area represents $80 \%$ of the stars. For most stars, the fraction of clean pixels reaches $90 \%$ at $10^{\prime \prime}$.

dwarfs. Table 8 lists them all, with their R.A., decl., associated host star, limiting magnitude in $z^{\prime}$ and $J$, apparent magnitude in [3.6] and [4.5], separation in au from the host star, and distance of the host star in pc. These unknown objects are possibly Ultra Luminous Galaxies (ULIRGS). ULIRGS are identified by their red [3.6]-[4.5] $>0.5$ colors meaning that they share colors with T dwarfs. Daddi et al. (2007) have shown that ULIRGS from the GOODS sample, with $0.7<z<1.3$, have a space density of $2 \times 10^{-5} \mathrm{Mpc}^{-3}$. At a luminosity distance corresponding to a redshift of $z=1$, about three ULIRGS should have been found per Spitzer/IRAC field. As ULIRGS have $F_{\nu} \sim 10 \mu J y$ for $z \sim 1$ to 2 (Kirkpatrick et al. 2012b), they are expected to be detected in our images.

A proper motion follow-up was obtained for all four candidates identified through their $z^{\prime}-J$ and [3.6]-[4.5] colors. It was carried out between 2016 and 2017, both at CFHT and at Gemini-South. Table 9 lists the candidates with their R.A., decl., host stars, $M_{z^{\prime}}, M_{J}, M_{3.6}, M_{4.5}$, separation in $\mathrm{au}$, pmra, pmdec, and the number of sigma at which the proper motion of the candidate differ from the host star's proper motion. The candidates are rejected at $3 \sigma$ or higher.

\section{Analysis and Discussion \\ 4.1. Sensitivity and Completeness}

For each image of our survey, the sensitivity to planets of a given semimajor axis and mass can be determined using the limiting magnitude reached as a function of the projected separations from the star and the corresponding fraction of pixels where a companion could have been detected. In computing these detection completeness maps for all stars in our sample, we adopted an approach similar to that of Nielsen et al. (2008) and Naud et al. (2017), relying on a Monte Carlo simulation.

First, for a given image and a given separation from the star, the fraction of clean pixels, i.e., pixels where a companion could have been detected if indeed it were present, was simply determined by counting pixels at that separation that were not flagged as bad, not saturated, and not affected by the presence of a star. Figures 11 and 12 show this fraction as a function of separation from the star for the $J$-band images and the [4.5]band images, respectively. In most cases, at $10^{\prime \prime}$ the fraction reaches 0.9 for the $J$-band images and 0.98 for the [4.5]-band

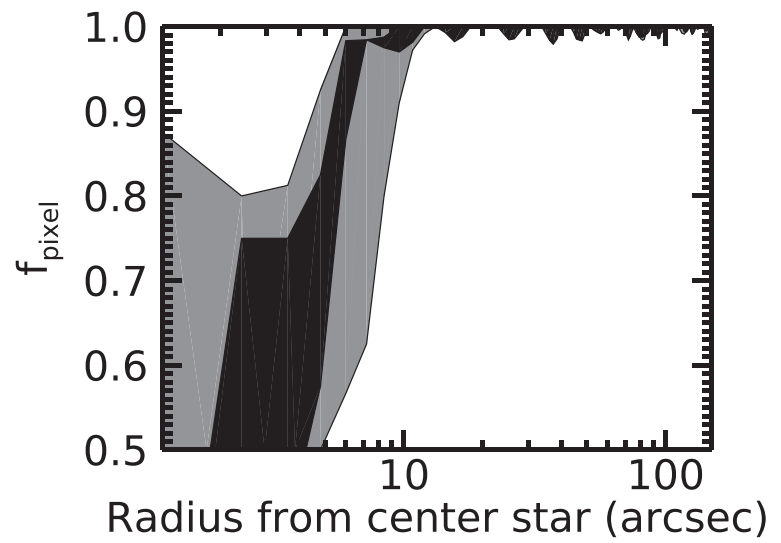

Figure 12. Same as 11 for Spitzer/IRAC observations at [4.5]. For most stars, the fraction of clean pixels reaches $98 \%$ at $10^{\prime \prime}$.

Table 10

Parameterization of the $J$-band Images Fraction of Pixel as a Logistic Function

\begin{tabular}{lccc}
\hline \hline Name & $a_{0}$ & $a_{1}$ & $a_{2}$ \\
\hline HIP 490 & 8.56 & 0.83 & 0.0331 \\
HIP 560 & 13.15 & 1.09 & 0.0777 \\
HIP 1113 & 25.65 & 5.59 & 0.0308 \\
HIP 1134 & -0.03 & 0.16 & 0.0131 \\
HIP 1481 & 9.54 & 0.86 & 0.0308 \\
\hline
\end{tabular}

(This table is available in its entirety in machine-readable form.)

Table 11

Parameterization of Spitzer's Fraction of Pixels as a Logistic Function

\begin{tabular}{lccc}
\hline \hline Name & $a_{0}$ & $a_{1}$ & $a_{2}$ \\
\hline HIP 490 & 1.91 & 0.45 & 0.0003 \\
\hline HIP 560 & -0.88 & 0.14 & -0.0013 \\
\hline HIP 1113 & 5.30 & 0.98 & 0.0030 \\
\hline HIP 1134 & 0.25 & 0.30 & -0.0014 \\
\hline HIP 1481 & 1.36 & 0.34 & -0.0005 \\
\hline
\end{tabular}

(This table is available in its entirety in machine-readable form.)

images. In a few cases, the target star is in the galactic plane, making the detection of a companion harder and the fraction lower. Huge variations in $f_{\text {pixel }}$ at smaller separations come from the different magnitude of the central stars, and the associated different areas affected by saturation. Some stars of the sample are very saturated, and thus $f_{\text {pixel }}$ is very low at small separation. On the other hand, the $\mathbf{M}$ dwarfs of our sample are not saturated, and thus a higher $f_{\text {pixel }}$ is reached at smaller separations. In general, the fraction of pixel for an individual target can be fitted by a logistic function with the shape of $1 /\left(e^{-a_{0}\left(x-a_{1}\right)}+e^{a_{2}}\right)$, where $a_{0}$ is the steepness of the curve, $a_{1}$ is the $x$-values of the midpoint, and $a_{2}$ is typically close to 0 . Tables 10 and 11 . show the values of the three parameters for each target of the sample for the $J$-band and [4.5] images respectively ( $a_{0}$ varies from -10 to $40, a_{1}$ goes from 0 to 14 , and $a_{2}$ is close to 0 ).

Next, we defined a grid of masses and semimajor axes, with the masses equally spaced in logarithmic scale between 0.5 and 

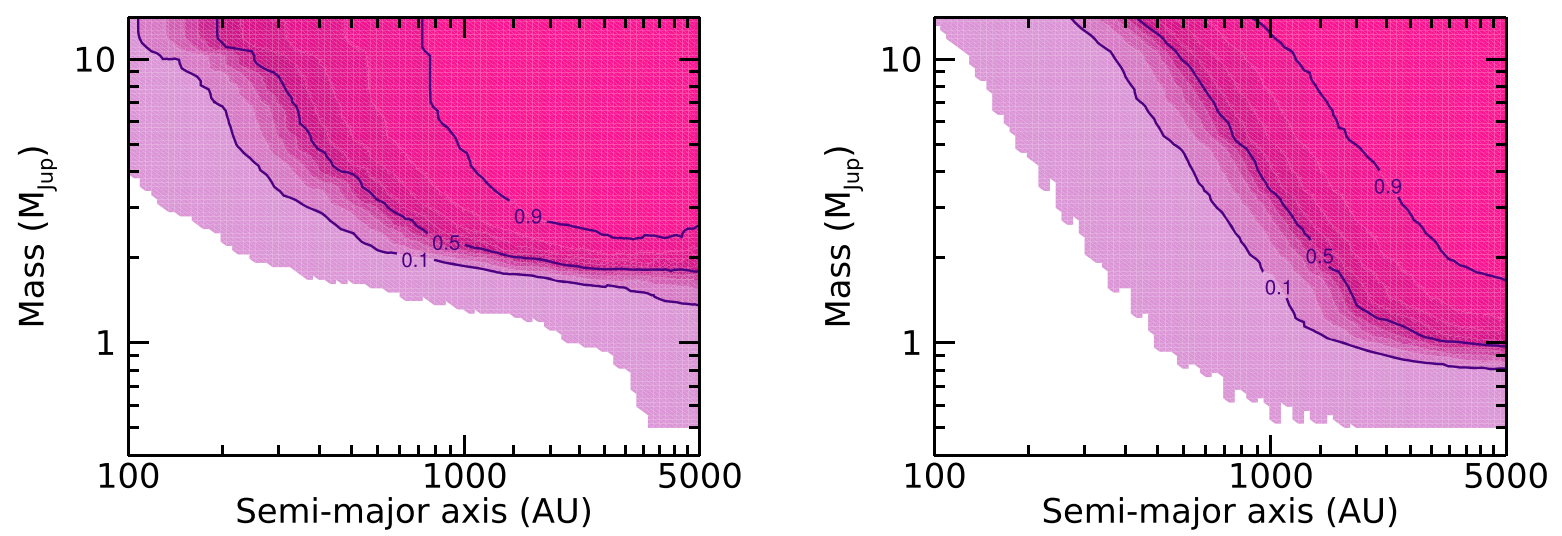

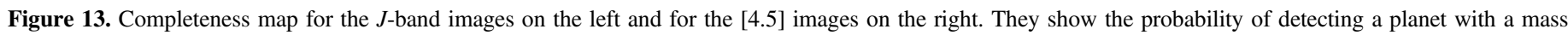
between 1 and $13 M_{\text {Jup }}$ as a function of the separation from the host star. Curves for $10 \%, 50 \%$, and $90 \%$ are shown.

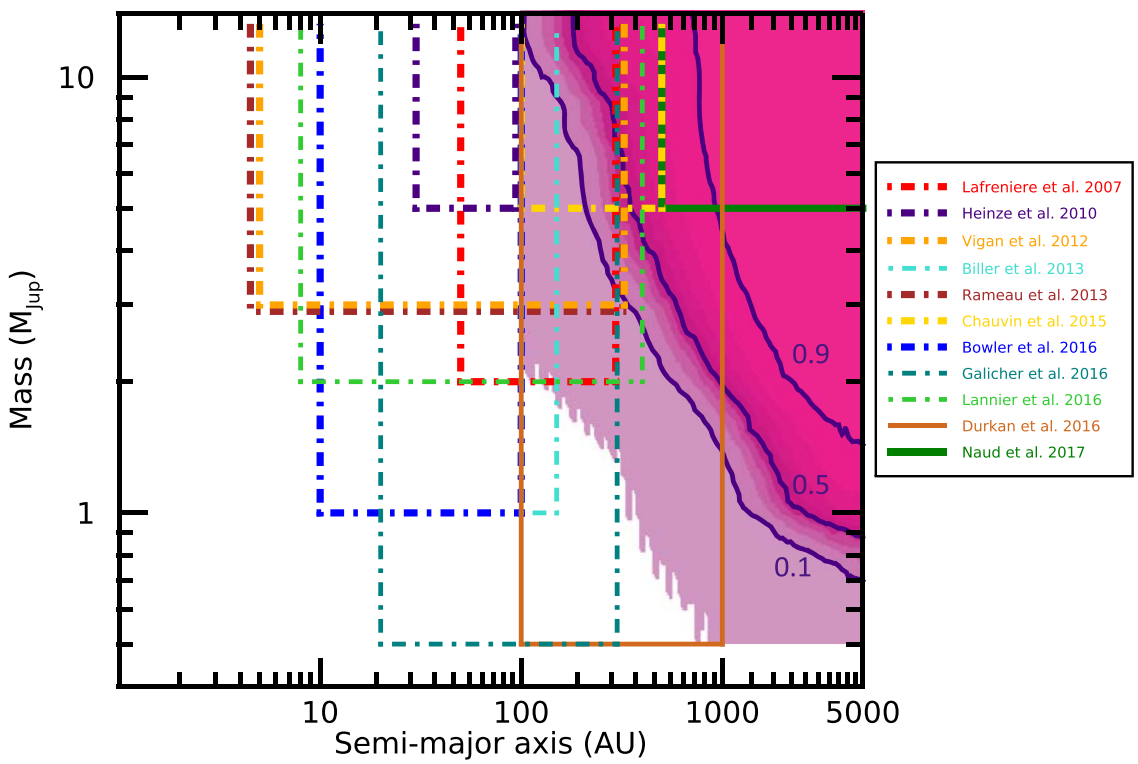

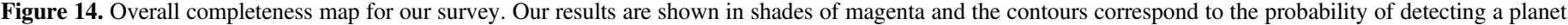

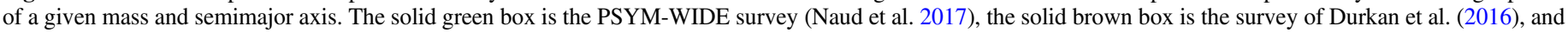

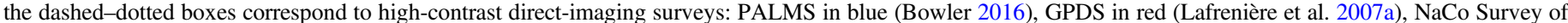

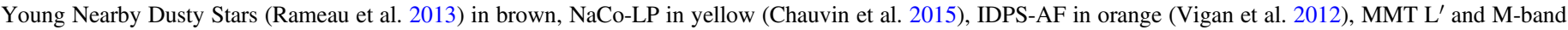

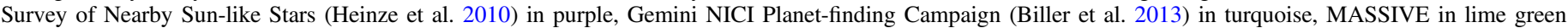

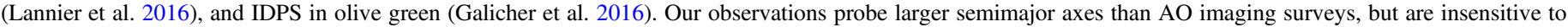
semimajor axes where AO observations are mostly sensitive.

$15 M_{\text {Jup }}$ and the semimajor axes equally spaced in logarithmic scale between 100 and $5000 \mathrm{au}$. For each point of the grid, we simulated $10^{4}$ planets. Each planet has an eccentricity taken randomly from the eccentricity distribution reported in Kipping (2013), which in turn is taken from the eccentricity from RV planets. Next, we used the method of Brandeker et al. (2006) and Brandt et al. (2014) to find the instantaneous projected separation of each planet, given their eccentricity, semimajor axis, and some random inclination and time of observation. The projected separation in au was finally converted to a projected angular separation in arcsec by dividing by the star distance, which is sampled uniformly within its interval of uncertainty.

For each grid point, we converted the mass into a $J$-band absolute magnitude using the AMES-Cond evolution models (Baraffe et al. 2003) and the ages of the targets from Tables 1 and 3 . We randomly sampled the age of each generated planet uniformly between the uncertainties given for the appropriate moving group (see Table 1). We then used the known distance of the star to convert the planets' absolute magnitudes to apparent magnitudes, and compared these to the detection limits found earlier to assess the detectability of each planet. If a planet was brighter than the detection limit, we used the fraction of clean pixels found earlier at that separation as the detection probability; otherwise the planet was assigned a detection probability of zero. This was repeated for each simulated planet, and the results were averaged to find the probability of detection at each point of the grid.

This procedure was repeated for all targets of the sample. The sensitivity of the whole survey was calculated by taking the median of all the detection probability maps. Two completeness maps were made this way, one for the $J$-band images (Figure 13, left) and one for the [4.5] band images (Figure 13, right). The ground-based survey is mostly sensitive to objects with masses higher than $2 M_{\text {Jup }}$ with a semimajor axis of more than $1000 \mathrm{au}$, while the Spitzer survey is sensitive to planets slightly less massive (down to $1 M_{\mathrm{Jup}}$ ) at larger separations. 


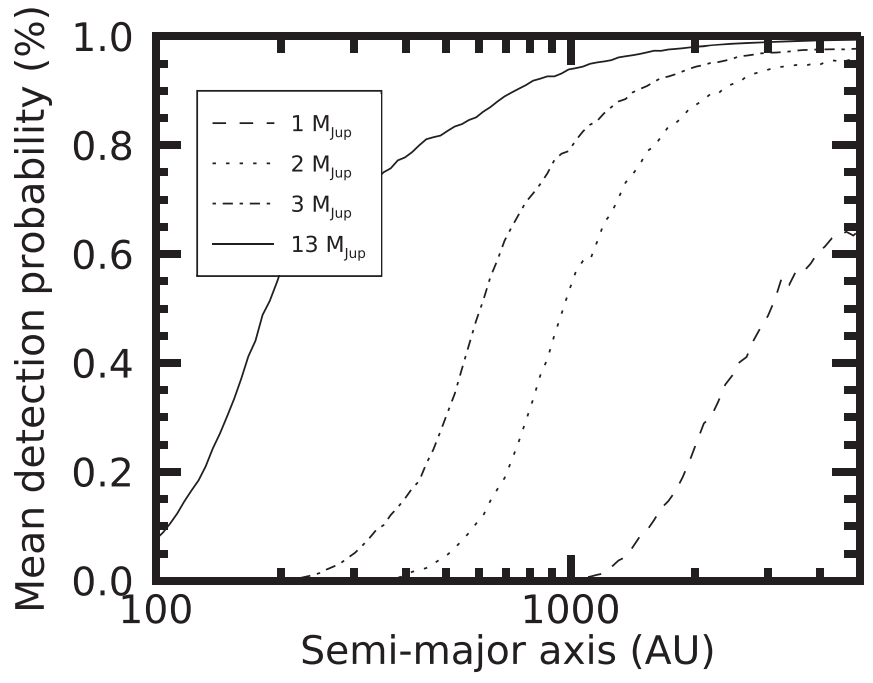

Figure 15. Mean detection probability for $1 M_{\mathrm{Jup}}(\mathrm{dash}), 2 M_{\mathrm{Jup}}(\mathrm{dot}), 3 M_{\mathrm{Jup}}$ (dash-dot), and $13 M_{\mathrm{Jup}}$ (solid) companions as a function of the semimajor axis in au.

The completeness maps for each star of the sample and for both $J$ and [4.5] bands were combined to build the overall completeness map of the survey. For each star at each point of the grid, the highest probability was taken between the completeness map of the $J$-band images and the [4.5] images. The two-band combined completeness maps were then averaged over all stars to obtain the overall survey completeness maps; see Figure 14. Figure 15 shows the mean detection probability as a function of semimajor axis for planetary objects with masses of $1 M_{\text {Jup }}, 2 M_{\text {Jup }}, 3 M_{\text {Jup }}$, and $13 M_{\text {Jup }}$, taken from the overall completeness map of the survey. The maximal probabilities of detection are respectively $64 \%, 95 \%$, $98 \%$, and $99 \%$. Our survey is mostly sensitive to planets with masses of $2 M_{\text {Jup }}$ and above, as the detection probability falls very rapidly between 2 and $1 M_{\text {Jup }}$.

Our results probe an area of the semimajor axis-mass diagram that has not been studied before. Figure 14 shows our completeness map compared to the regions probed by the following other studies: the PSYM-WIDE survey (Naud et al. 2017), aimed at discovering planetary mass objects on wide orbits around K5-L5 dwarfs; the PALMS survey (Bowler 2016), a deep coronagraphic study of 78 single young nearby $(<40 \mathrm{pc}) \mathrm{M}$ dwarfs; the GPDS survey (Lafrenière et al. 2007a), a survey of young stars searching for giant planets on large orbits; the NaCo Survey of Young Nearby Dusty Stars (Rameau et al. 2013), which targeted 59 young nearby AFGK stars; the NaCo-LP survey (Chauvin et al. 2015), which focused on 86 young, bright, and primarily FGK stars; the IDPS-AF survey (Vigan et al. 2012), which observed 42 AF stars, the MMT L' and M-band Survey of 54 nearby FGK stars (Heinze et al. 2010); the Gemini NICI Planet-finding Campaign (Biller et al. 2013), which targeted 230 young stars of all spectral types, MASSIVE (Lannier et al. 2016); which targeted 58 young and nearby M-type dwarfs; the IDPS survey Galicher et al. (2016), which combines results for 292 young nearby stars; and Durkan et al. (2016), who studied 121 nearby stars observed with SPITZER/IRAC. On the whole, this survey is a good complement to AO imaging surveys, being mostly sensitive at separations of several hundreds of au but insensitive at semimajor axes of less than $\sim 150 \mathrm{au}$, where AO imaging surveys are most sensitive.

\subsection{Constraints on Additional Companions in Systems with Known Directly Imaged Companions}

At least one planetary-mass or brown dwarf companion was previously found around six stars in our sample; most of these companions were found using high-contrast AO imaging. Our search, being sensitive to much wider separations and reaching lower masses, adds valuable constraints on the presence of additional companions in these systems. In Figure 16, we provide the individual completeness maps from our survey for these six systems.

The companion $\mathrm{Pz}$ Tel B, a $36 \pm 6 M_{\text {Jup }}$ brown dwarf orbiting at $16.4 \pm 1$ au from a pre-main sequence G9 star member of the $\beta$-Pictoris association, was found by Biller et al. (2010) using VLT/NACO. We put constraints on the presence of companions at larger orbits (see Figure 16, top left). At a confidence level of more than $90 \%$, we can reject a companion with masses as low as 1-2 $M_{\mathrm{Jup}}$ at 2000-5000 au.

The companion 2M1207 b, a $4 \pm 1 M_{\text {Jup }}$ object (Chauvin et al. 2004) orbiting at $46_{-15}^{+37}$ au (Blunt et al. 2017) around the young brown dwarf TWA27, a member of the TW Hydrae association at $52 \mathrm{pc}$, was discovered using VTL/NACO. Our survey put strong constraints on the presence of $>10 M_{\text {Jup }}$ objects in the system, as they should have been detected at separations from 100 to $5000 \mathrm{au}$. Moreover, at a distance of $1000 \mathrm{au}$, the detection probability of a $1 M_{\text {Jup }}$ object is about $80 \%$. Our survey covers quite well the regime of separations $>1000$ au and masses $>1 M_{\text {Jup }}$ (see Figure 16 , top right). No companion was detected by our survey.

Chauvin et al. (2005) found a $13.5 \pm 0.5 M_{\text {Jup }}$ object at $250 \mathrm{au}$ of $\mathrm{AB}$ Pic, a K2V star member of the TucanaHorologium association, by using VLT/NACO. Figure 16, middle left, presents the completeness reached by our survey. We put strong constraints on the presence of companions of $2 M_{\text {Jup }}$ or more at separations higher than $1000 \mathrm{au}$.

Marois et al. $(2008,2010)$ used AO observations with Keck/ NIRC2 and Gemini/NIRI to find four planets of $7_{-2}^{+4}, 10_{-3}^{+3}$, $10_{-3}^{+3}$, and $9_{-4}^{+4} M_{\text {Jup }}$ at respectively $\sim 68,43,27$, and 17 au from HR 8799 (Wertz et al. 2017), an A5V star member of the Columba association. We probed a region in mass that is equivalent to the planets already known, but at much larger semimajor axes. We put good constraints on the presence of companion with $\geqslant 4 M_{\text {Jup }}$ and semimajor axis greater than $1500 \mathrm{au}$.

Lagrange et al. (2009) found a $12.7 \pm 0.3 M_{\text {Jup }}$ (Morzinski et al. 2015) planet at 9.2 $2_{0.4}^{+1.5}$ au (Millar-Blanchaer et al. 2015) orbiting $\beta$ Pictoris, an $\mathrm{A} 6 \mathrm{~V}$ star member of the $\beta$ Pictoris association, using high-contrast VLT/NACO observations. Our observations put strong constraints on the existence of objects of $1 M_{\mathrm{Jup}}$ or more at semimajor axes of $>1000 \mathrm{au}$.

A 1-2 $M_{\text {Jup }}{ }^{8}$ (Rajan et al. 2017) object orbiting 51 Eri at $\sim 14$ au, an F0IV star, was found by Macintosh et al. (2015) using Gemini/GPI. 51 Eri is part of a triple system, bound to and separated by $\sim 2000$ au from GJ3305AB, an $\mathrm{M}+\mathrm{M}$ binary of unresolved spectral type M0 (Montet et al. 2015). Our survey put strong constraints on the presence of companions of mass $>1 M_{\text {Jup }}$ at semimajor axes between 100 and $5000 \mathrm{au}$.

\footnotetext{
This mass was inferred from a hot start model from (Marley et al. 2007). It is also possible that the mass is anywhere between 2 and $12 M_{\text {Jup }}$, according to the cold start model from Fortney et al. (2008).
} 

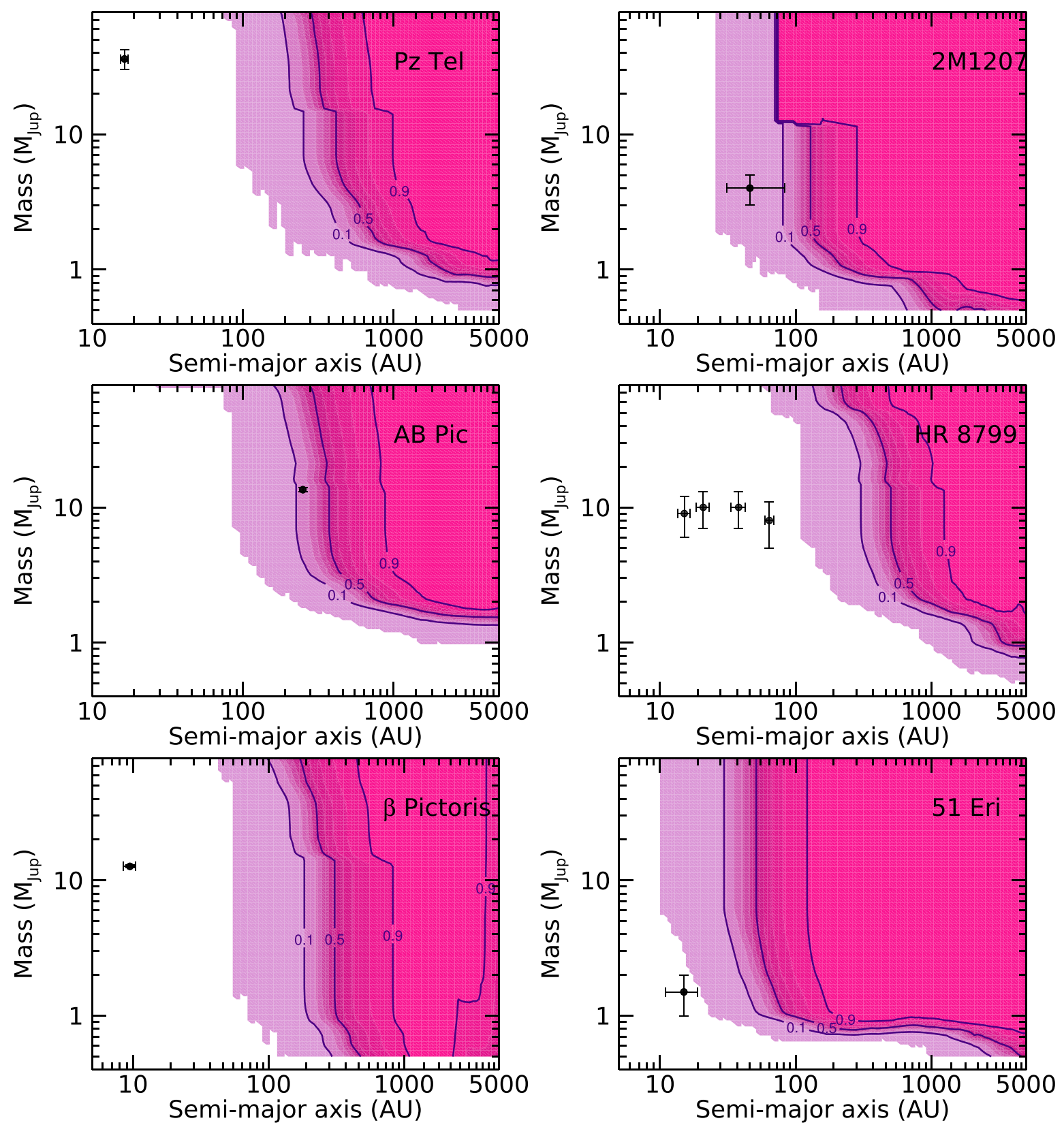

Figure 16. Contrast curves for Pz Tel, 2M1207, AB Pic, HR 8799, $\beta$ Pictoris, and 51 Eri. Known companions are shown as black points with error bars, using masses from hot start models. See text for references for the masses.

\subsection{Planet Frequency}

Based on the null result of our survey, and our completeness limits calculated in Section 4.2, we evaluated an upper limit to the frequency of occurrence of planets at large semimajor axis (1000-5000 au), following the method developed by Lafrenière et al. (2007a).

If we have $N=177$ stars, enumerated from $j=1$ to $N$, and we survey an interval of mass going from 1 to $13 M_{\mathrm{Jup}}$ and an interval of semimajor axis of 1000-5000 au, then we define $f$ to be the fraction of stars with at least one companion in the intervals and $p_{j}$ the probability of detecting such a companion. This probability is computed from the completeness map calculated previously by taking the mean of the probability at each point of the $100 \times 100$ grid. Given that the grid is uniform in logarithmic space, this amounts to assuming that the semimajor axis and the mass are distributed uniformly in $\log$.
The detections in the survey are characterized by the set $\left\{d_{j}\right\}$, and in our case, because the survey gave a null result (all known companions around our targets were too close-in to be seen in our data), $d_{j}=0$ for all $j$. The probability of observing the set $\left\{d_{j}\right\}$ in our survey is given by the following binomial likelihood,

$$
\mathcal{L}\left(\left\{d_{j}\right\} \mid f\right)=\prod_{j=0}^{N}\left(1-f p_{j}\right)^{1-d_{j}}\left(f p_{j}\right)^{d_{j}} .
$$

Then according to Bayes' theorem, the posterior distribution for $f$, in light of our results, is given by

$$
p\left(f \mid\left\{d_{j}\right\}\right)=\frac{\mathcal{L}\left(\left\{d_{j}\right\} \mid f\right) p(f)}{\int_{0}^{1} \mathcal{L}\left(\left\{d_{j}\right\} \mid f\right) p(f) \mathrm{df}},
$$


where $p(f)$ is the prior probability on $f$, reflecting our state of knowledge independent of our new data. One has to be careful in the choice of the prior; here, we elected to use a noninformative Jeffrey's prior (see Berger et al. 2009), given by

$$
P(f)=\frac{1}{\pi} \frac{1}{\sqrt{f}} \frac{1}{\sqrt{1-f}} .
$$

For our survey with no detection, the posterior distribution of $f$ peaks at 0 , and we can only set an upper limit on $f$ (by integrating the posterior from 0 to the fraction $f$ that gives a probability matching the desired confidence level).

We obtained an upper limit for the fraction of stars with at least one planet of $f_{\max }=0.03$ at a $95 \%$ confidence level, for planets with masses between 1 and $13 M_{\text {Jup }}$ and semimajor axis between 1000 and 5000 au distributed uniformly in $\log$ space.

\section{Conclusions}

A sample of 177 young stars, all bona fide members of moving groups, were observed between 2014B and 2017B by CFHT's MegaCam in the $z_{a b}^{\prime}$-band and WIRCam in the $J$-band, or Gemini GMOS-S in the $z_{a b}^{\prime}$-band and Flamingos-2 in the $J$-band, as well as with Spitzer/IRAC at [3.6] and [4.5] to search for planetary-mass companions on very wide orbits (up to $5000 \mathrm{au}$ ). The survey made use of the very red $z^{\prime}-J$ and [3.6]-[4.5] colors intrinsic to such objects and reached good sensitivities down to objects of $1 M_{\text {Jup }}$. Four candidates were identified through colors selection, but proper motion followup obtained a year after the first epoch rejected the candidates. No planet was found. This null result allowed us to set an upper limit of 0.03 for the fraction of stars with at least one planet with mass between 1 and $13 M_{\text {Jup }}$ and semimajor axis between 1000 and $5000 \mathrm{au}$, at a 95\% confidence level, assuming logarithmically uniform distributions in planet mass and semimajor axis. While it was not the main objective of the survey, our data also constrain the frequency of brown dwarfs to be less than $2.2 \%$ for objects with masses between 13 and $80 M_{\text {Jup }}$ and for semimajor axis between 1000 and $5000 \mathrm{au}$.

As mentioned above, the formation process by which Jupiter-like objects on wide orbits form has been the subject of an ongoing debate. The very low occurrence rate for planets at 1000-5000 au found by our survey indicates that neither core accretion nor disk instability is actually efficient at forming gas giants at these large separations. It is possible that the few known instances of planets at such large separations from their host star represent the low-mass tail end of distribution of brown dwarf companions that form like stars, rather than objects that form like planets. More quantitative implications of our results on the properties of the overall distribution of planets around stars, as well as on the formation mechanism of very distant companions, will be explored in a forthcoming paper, where we will further incorporate the results of $\mathrm{AO}$ surveys.

Based on observations obtained at the Gemini Observatory through programs number GS-2014B-Q-2, GS-2015A-Q-71, GS-2015B-Q-57, GS-2016A-Q-69, GS-2016B-Q-33, GS2017A-Q-58, and GS-2017B-Q-34. The Gemini Observatory is operated by the Association of Universities for Research in Astronomy, Inc., under a cooperative agreement with the National Science Foundation (NSF) on behalf of the Gemini partnership: the NSF (United States), the National Research Council (Canada), CONICYT (Chile), the Australian Research Council (Australia), Ministério da Ciência, Tecnologia e Inovação (Brazil), and Ministerio de Ciencia, Tecnología e Innovación Productiva (Argentina).

The authors would like to thank the anonymous referee for constructive comments and suggestions that improved the overall quality of the paper. This work was financially supported by the Fond de Recherche Québécois-Nature et Technologie(FRQNT; Québec).

Based on observations obtained with MegaPrime/MegaCam, a joint project of CFHT and CEA/DAPNIA, at the Canada-France-Hawaii Telescope (CFHT) which is operated by the National Research Council (NRC) of Canada, the Institut National des Science de l'Univers of the Centre National de la Recherche Scientifique (CNRS) of France, and the University of Hawaii.

The Pan-STARRS1 Surveys (PS1) have been made possible through contributions of the Institute for Astronomy, the University of Hawaii, the Pan-STARRS Project Office, the Max-Planck Society and its participating institutes, the Max Planck Institute for Astronomy, Heidelberg, and the Max Planck Institute for Extraterrestrial Physics, Garching, The Johns Hopkins University, Durham University, the University of Edinburgh, Queen's University Belfast, the HarvardSmithsonian Center for Astrophysics, the Las Cumbres Observatory Global Telescope Network Incorporated, the National Central University of Taiwan, the Space Telescope Science Institute, the National Aeronautics and Space Administration under Grant No. NNX08AR22G issued through the Planetary Science Division of the NASA Science Mission Directorate, the National Science Foundation under Grant No. AST-1238877, the University of Maryland, and Eotvos Lorand University (ELTE) and the Los Alamos National Laboratory.

This work has made use of data from the European Space Agency (ESA) mission Gaia (https://www.cosmos.esa.int/ gaia), processed by the Gaia Data Processing and Analysis Consortium (DPAC, https://www.cosmos.esa.int/web/gaia/ dpac/consortium). Funding for the DPAC has been provided by national institutions, particularly those participating in the Gaia Multilateral Agreement.

Facilities: Gemini-South (Flamingos-2, GMOS-S), CFHT (WIRCam, MegaCam), Spitzer (Irac).

Software: SExtractor (Bertin \& Arnouts 1996), Scamp (Bertin 2010a), Swarp (Bertin 2010b), CFHT'S Elixir pipeline.

\section{ORCID iDs}

Étienne Artigau (iD https://orcid.org/0000-0003-3506-5667 Julien Rameau (10 https://orcid.org/0000-0003-0029-0258 David Lafrenière (iD https://orcid.org/0000-0002-6780-4252 Jonathan Gagné (iD https://orcid.org/0000-0002-2592-9612 Loïc Albert (iD https://orcid.org/0000-0003-0475-9375 Marie-Eve Naud (i) https://orcid.org/0000-0003-1807-1598 Markus Janson (i) https://orcid.org/0000-0001-8345-593X

\section{References}

Ahn, C. P., Alexandroff, R., Allende Prieto, C., et al. 2012, ApJS, 203, 21 Albert, L. 2006, PhD thesis, Université de Montréal

Albert, L., Artigau, É., Delorme, P., et al. 2011, AJ, 141, 203

Alibert, Y., Pont, F., Baraffe, I., et al. 2009, A\&A, 506, 391

Baraffe, I., Chabrier, G., Barman, T. S., Allard, F., \& Hauschildt, P. H. 2003, A\&A, 402, 701 
Baruteau, C., \& Masset, F. 2013, Lecture Notes in Physics, Vol. 861 (Berlin: Springer), 201

Bate, M. R. 2012, MNRAS, 419, 3115

Bate, M. R., Bonnell, I. A., \& Bromm, V. 2002, MNRAS, 332, L65

Beichman, C., Gelino, C. R., Kirkpatrick, J. D., et al. 2014, ApJ, 783, 68

Bell, C. P. M., Mamajek, E. E., \& Naylor, T. 2016, in IAU Symp. 314, Young Stars \& Planets Near the Sun (Cambridge: Cambridge Univ. Press), 41

Berger, J. O., Bernardo, J. M., \& Sun, D. 2009, The Annals of Statistics, 37, 905

Bertin, E. 2010a, Scamp, Astrophysics Source Code Library, ascl:1010.063

Bertin, E. 2010b, Swarp, Astrophysics Source Code Library, ascl:1010.068

Bertin, E., \& Arnouts, S. 1996, A\&AS, 117, 393

Biller, B. A., Liu, M. C., Wahhaj, Z., et al. 2010, ApJL, 720, L82

Biller, B. A., Liu, M. C., Wahhaj, Z., et al. 2013, ApJ, 777, 160

Binks, A. S., Jeffries, R. D., \& Maxted, P. F. L. 2015, MNRAS, 452, 173

Blunt, S., Nielsen, E. L., Rosa, D., et al. 2017, AJ, 153, 229

Boss, A. P. 2011, ApJ, 731, 74

Boulade, O., Vigroux, L. G., Charlot, X., et al. 1998, Proc. SPIE, 3355, 614

Bowler, B. P. 2016, PASP, 128, 102001

Bowler, B. P., Liu, M. C., Shkolnik, E. L., \& Tamura, M. 2015, ApJS, 216, 7

Brandeker, A., Jayawardhana, R., Khavari, P., Haisch, K. E., Jr., \& Mardones, D. 2006, ApJ, 652, 1572

Brandt, T. D., McElwain, M. W., Turner, E. L., et al. 2014, ApJ, 794, 159

Bryan, M. L., Bowler, B. P., Knutson, H. A., et al. 2016, ApJ, 827, 100

Chambers, K., \& Team, P.-S. 2018, AAS Meeting, 231, 102.01

Chatterjee, S., Ford, E. B., Matsumura, S., \& Rasio, F. A. 2008, ApJ, 686, 580

Chauvin, G., Lagrange, A.-M., Dumas, C., et al. 2004, A\&A, 425, L29

Chauvin, G., Lagrange, A.-M., Zuckerman, B., et al. 2005, A\&A, 438, L29

Chauvin, G., Vigan, A., Bonnefoy, M., et al. 2015, A\&A, 573, A127

Choquet, É., Pueyo, L., Soummer, R., et al. 2015, Proc. SPIE, 9605, 96051P

Cutri, R. M., Skrutskie, M. F., van Dyk, S., et al. 2003, yCat, 2246, 0

Daddi, E., Dickinson, M., Morrison, G., et al. 2007, ApJ, 670, 156

de la Reza, R., Torres, C. A. O., Quast, G., Castilho, B. V., \& Vieira, G. L. 1989, ApJL, 343, L61

Delorme, P., Albert, L., Forveille, T., et al. 2010, A\&A, 518, A39

Delorme, P., Delfosse, X., Albert, L., et al. 2008, A\&A, 482, 961

Delorme, P., Gagné, J., Girard, J. H., et al. 2013, A\&A, 553, L5

Dupuy, T. J., \& Liu, M. C. 2012, ApJS, 201, 19

Durkan, S., Janson, M., \& Carson, J. C. 2016, ApJ, 824, 58

Eikenberry, S., Bandyopadhyay, R., Bennett, J. G., et al. 2012, Proc. SPIE, 8446, 84460I

Faherty, J. K., Riedel, A. R., Cruz, K. L., et al. 2016, ApJS, 225, 10

Fazio, G. G., Hora, J. L., Allen, L. E., et al. 2004, ApJS, 154, 10

Fortney, J. J., Marley, M. S., Saumon, D., \& Lodders, K. 2008, ApJ, 683, 1104

Gagné, J., Allers, K. N., Theissen, C. A., et al. 2018a, ApJL, 854, L27

Gagné, J., Burgasser, A. J., Faherty, J. K., et al. 2015a, ApJL, 808, L20

Gagné, J., Faherty, J. K., Cruz, K. L., et al. 2015b, ApJS, 219, 33

Gagné, J., Lafrenière, D., Doyon, R., Malo, L., \& Artigau, É 2014, ApJ, 783,121

Gagné, J., Mamajek, E. E., Malo, L., et al. 2018b, ApJ, 856, 23

Gaia Collaboration, Brown, A. G. A., Vallenari, A., et al. 2018, arXiv:1804. 09365

Gaia Collaboration, Prusti, T., de Bruijne, J. H. J., et al. 2016, A\&A, 595, A1

Galicher, R., Marois, C., Macintosh, B., et al. 2016, A\&A, 594, A63

Gauza, B., Béjar, V. J. S., Pérez-Garrido, A., et al. 2015, ApJ, 804, 96

Gimeno, G., Roth, K., Chiboucas, K., et al. 2016, Proc. SPIE, 9908, 99082S

Goldman, B., Marsat, S., Henning, T., Clemens, C., \& Greiner, J. 2010, MNRAS, 405, 1140

Hawley, S. L., Covey, K. R., Knapp, G. R., et al. 2002, AJ, 123, 3409

Heinze, A. N., Hinz, P. M., Kenworthy, M., et al. 2010, ApJ, 714, 1570

Hook, I. M., Jørgensen, I., Allington-Smith, J. R., et al. 2004, PASP, 116, 00290,425

Inaba, S., Wetherill, G. W., \& Ikoma, M. 2003, Icar, 166, 46

Janson, M., Bonavita, M., Klahr, H., et al. 2011, ApJ, 736, 89

Janson, M., Brandt, T. D., Moro-Martín, A., et al. 2013, ApJ, 773, 73

Janson, M., Quanz, S. P., Carson, J. C., et al. 2015, A\&A, 574, A120

Kastner, J. H., Zuckerman, B., Weintraub, D. A., \& Forveille, T. 1997, Sci, 277, 67

Kipping, D. M. 2013, MNRAS, 434, L51

Kirkpatrick, A., Pope, A., Alexander, D. M., et al. 2012a, ApJ, 759, 139

Kirkpatrick, J. D., Gelino, C. R., Cushing, M. C., et al. 2012b, ApJ, 753, 156

Kiss, L. L., Moór, A., Szalai, T., et al. 2011, MNRAS, 411, 117

Kraus, A. L., Shkolnik, E. L., Allers, K. N., \& Liu, M. C. 2014, AJ, 147, 146

Lafrenière, D., Doyon, R., Marois, C., et al. 2007a, ApJ, 670, 1367

Lafrenière, D., Marois, C., Doyon, R., Nadeau, D., \& Artigau, É 2007b, ApJ, 660,770

Lagrange, A.-M., Gratadour, D., Chauvin, G., et al. 2009, A\&A, 493, L21
Lannier, J., Delorme, P., Lagrange, A. M., et al. 2016, A\&A, 596, A83 Lépine, S., \& Simon, M. 2009, AJ, 137, 3632

Lindegren, L., Hernandez, J., Bombrun, A., et al. 2018, arXiv:1804.09366

Liu, M. C., Magnier, E. A., Deacon, N. R., et al. 2013, ApJL, 777, L20

Lodieu, N., Béjar, V. J. S., \& Rebolo, R. 2013, A\&A, 550, L2

Looper, D. L., Bochanski, J. J., Burgasser, A. J., et al. 2010, AJ, 140, 1486

Macintosh, B., Graham, J. R., Barman, T., et al. 2015, Sci, 350, 64

Makarov, V. V., \& Urban, S. 2000, MNRAS, 317, 289

Malo, L., Doyon, R., Feiden, G. A., et al. 2014, ApJ, 792, 37

Malo, L., Doyon, R., Lafrenière, D., et al. 2013, ApJ, 762, 88

Marley, M. S., Fortney, J. J., Hubickyj, O., Bodenheimer, P., \& Lissauer, J. J. 2007, ApJ, 655, 541

Marois, C., Macintosh, B., Barman, T., et al. 2008, Sci, 322, 1348

Marois, C., Zuckerman, B., Konopacky, Q. M., Macintosh, B., \& Barman, T. 2010, Natur, 468, 1080

Mayor, M., \& Queloz, D. 1995, Natur, 378, 355

McMahon, R. G., Banerji, M., Gonzalez, E., et al. 2013, Msngr, 154, 35

Millar-Blanchaer, M. A., Graham, J. R., Pueyo, L., et al. 2015, ApJ, 811, 18

Montet, B. T., Bowler, B. P., Shkolnik, E. L., et al. 2015, ApJL, 813, L11

Moór, A., Szabó, G. M., Kiss, L. L., et al. 2013, MNRAS, 435, 1376

Mordasini, C., Alibert, Y., Klahr, H., \& Henning, T. 2012, A\&A, 547, A111

Morzinski, K. M., Males, J. R., Skemer, A. J., et al. 2015, ApJ, 815, 108

Naud, M.-E., Artigau, É., Doyon, R., et al. 2017, AJ, 154, 129

Naud, M.-E., Artigau, É, Malo, L., et al. 2014, ApJ, 787, 5

Nayakshin, S. 2017, MNRAS, 470, 2387

Nielsen, E. L., Close, L. M., Biller, B. A., Masciadri, E., \& Lenzen, R. 2008, ApJ, 674, 466

Nielsen, E. L., Liu, M. C., Wahhaj, Z., et al. 2013, ApJ, 776, 4

Padoan, P., \& Nordlund, А 2004, ApJ, 617, 559

Pollack, J. B., Hubickyj, O., Bodenheimer, P., et al. 1996, Icar, 124, 62

Puget, P., Stadler, E., Doyon, R., et al. 2004, Ground-based Instrumentation for Astronomy, 5492, 978

Rajan, A., Rameau, J., de Rosa, R. J., et al. 2017, AJ, 154, 10

Rameau, J., Chauvin, G., Lagrange, A.-M., et al. 2013, A\&A, 553, A60

Riedel, A. R., Finch, C. T., Henry, T. J., et al. 2014, AJ, 147, 85

Rodriguez, D. R., Bessell, M. S., Zuckerman, B., \& Kastner, J. H. 2011, ApJ, 727,62

Rodriguez, D. R., Zuckerman, B., Kastner, J. H., et al. 2013, ApJ, 774, 101

Schlieder, J. E., Lépine, S., \& Simon, M. 2010, AJ, 140, 119

Schlieder, J. E., Lépine, S., \& Simon, M. 2012a, AJ, 143, 80

Schlieder, J. E., Lépine, S., \& Simon, M. 2012b, AJ, 144, 109

Schneider, G., Song, I., Zuckerman, B., et al. 2004, AAS Meeting, 205, 11.14

Shkolnik, E., Liu, M. C., \& Reid, I. N. 2009, ApJ, 699, 649

Shkolnik, E. L., Allers, K. N., Kraus, A. L., Liu, M. C., \& Flagg, L. 2017, AJ, 154, 69

Shkolnik, E. L., Anglada-Escudé, G., Liu, M. C., et al. 2012, ApJ, 758, 56

Shkolnik, E. L., Liu, M. C., Reid, I. N., Dupuy, T., \& Weinberger, A. J. 2011, ApJ, 727, 6

Smart, R. L., Marocco, F., Caballero, J. A., et al. 2017, MNRAS, 469, 401

Soderblom, D. R. 2010, ARA\&A, 48, 581

Song, I., Zuckerman, B., \& Bessell, M. S. 2003, ApJ, 599, 342

Soummer, R., Choquet, E., Pueyo, L., et al. 2016, AAS Meeting, 227, 137.03

Soummer, R., Pueyo, L., \& Larkin, J. 2012, ApJL, 755, L28

Stamatellos, D., Hubber, D. A., \& Whitworth, A. P. 2007, MNRAS, 382, L30

Tonry, J. L., Stubbs, C. W., Lykke, K. R., et al. 2012, ApJ, 750, 99

Torres, C. A. O., da Silva, L., Quast, G. R., de la Reza, R., \& Jilinski, E. 2000 AJ, 120, 1410

Torres, C. A. O., Quast, G. R., Melo, C. H. F., \& Sterzik, M. F. 2008, in Handbook of Star-forming Regions, Volume II: The Southern Sky ASP Monograph Publications, ed. B. Reipurth (San Francisco, CA: ASP), 757

Veras, D., Crepp, J. R., \& Ford, E. B. 2009, ApJ, 696, 1600

Vigan, A., Bonavita, M., Biller, B., et al. 2017, A\&A, 603, A3

Vigan, A., Patience, J., Marois, C., et al. 2012, A\&A, 544, A9

Wahhaj, Z., Liu, M. C., Nielsen, E. L., et al. 2013, ApJ, 773, 179

Wang, J., Fischer, D. A., Xie, J.-W., \& Ciardi, D. R. 2015, ApJ, 813, 130

Wertz, O., Absil, O., González, G., et al. 2017, A\&A, 598, A83

Wolf, C., Onken, C. A., Luvaul, L. C., et al. 2018, PASA, 35, e010

Zuckerman, B., Rhee, J. H., Song, I., \& Bessell, M. S. 2011, ApJ, 732, 61

Zuckerman, B., \& Song, I. 2004, ARA\&A, 42, 685

Zuckerman, B., Song, I., \& Bessell, M. S. 2004, ApJL, 613, L65

Zuckerman, B., Song, I., Bessell, M. S., \& Webb, R. A. 2001a, ApJL, 562, L87

Zuckerman, B., Song, I., \& Webb, R. A. 2001b, ApJ, 559, 388

Zuckerman, B., \& Webb, R. A. 2000, ApJ, 535, 959

Zuckerman, B. 2001, in ASP Conf. Ser. 244, Young Stars Near Earth: Progress and Prospects, ed. R. Jayawardhana \& T. Greene (San Francisco, CA: ASP), 122 